\title{
Remarks on Geo-Logarithmic Price Indices
}

\author{
Jacek Biatek ${ }^{1}$
}

\begin{abstract}
As is known, all geo-logarithmic indices enjoy the axiomatic properties of being proportional, commensurable and homogeneous, together with their cofactors (Martini 1992a). Geologarithmic price indices satisfying the axioms of monotonicity, basis reversibility and factor reversibility have been investigated by Marco Fattore (2010), who has shown that the superlative Fisher price index does not belong to this family of indices. In this article, we discuss geo-logarithmic price indices with reference to the Laspeyres-Paasche bounding test and we propose a modification of the considered index family that satisfies this test. We also modify the structure of geo-logarithmic indices by using an additional parameter and, following the economic approach, we list superlative price index formulas that are members of the considered price index family. We obtain a special subfamily that approximates superlative price indices and includes the Fisher, Walsh and Sato-Vartia price indices.
\end{abstract}

Key words: Price index theory; geo-logarithmic price indices; superlative indices.

\section{Introduction}

The literature on the axiomatic index theory is very extensive (Krstcha 1988; Balk 1995; Von der Lippe 2007). From a theoretical point of view, a well-constructed index should satisfy a group of postulates (tests) arising from the axiomatic index theory. A system of minimum requirements for an index comes from Martini (1992b). According to the abovementioned system, a price index should satisfy at least three conditions: identity, commensurability and linear homogeneity (see Appendix A, Subsection 8.1). German index theoreticians - Eichhorn and Voeller (1976) - introduced a more generally acceptable system (EV) of five, and later also of four, axioms: strict monotonicity, price dimensionality, commensurability, identity and (optionally) linear homogeneity. These five axioms imply other tests such as proportionality (identity plus linear homogeneity) or quantity dimensionality ( price dimensionality plus commensurability) - see Von der Lippe (2007). In the literature, we can also encounter other systems - for example Olt (1996) examined several systems that provide less restrictive requirements than EVsystems. Moreover, some authors consider general price index formulas as having the above-mentioned desirable properties (Diewert 1976; Hill 2006; Fattore 2010; Białek 2012).

${ }^{1}$ Department of Statistical Methods, University of Lodz, ul. Uniwersytecka 3, 90-137, Lodz, Poland. Email: jbialek@uni.lodz.pl.

Acknowledgments: The article is financed by the National Science Centre in Poland (grant no. 2017/25/B/HS4/00387). I would like to thank some anonymous reviewers for their very helpful comments and inspiring remarks. 
In this article, we discuss geo-logarithmic price indices, being a class of indices that contains several well-known indices and thus provides a useful framework for comparing properties of different index formulas (Fattore 2006, 2010). We analyse and modify geologarithmic price indices with reference to the Laspeyres-Paasche bounding test, which can be motivated in the 'economic approach'. According to this approach, upper and lower bounds for the index are provided by the Laspeyres and Paasche price index formulas. This follows from the choice of a cost of living index (COLI) as a target for the index, with an assumption about consumers' cost minimising behaviour. From the economic approach point of view, a "good" index should have a value between the above-mentioned bounds, that is, it should satisfy the Laspeyres-Paasche bounding test. We also modify the structure of geo-logarithmic indices by using an additional parameter and, following the economic approach, we list superlative price index formulas that are members of the considered price index family or obtained as the first-order approximation of the geologarithmic price index.

Our motivation has its genesis in the inflation measurement. The final report of the Boskin Commission begins with a recommendation that "the Bureau of Labour Statistics (BLS) should establish a cost of living index (COLI) as its objective in measuring consumer prices" (Boskin et al. 1996, 2). Further discussion on the theory of the COLI can be found in the following papers: Diewert (1993), Jorgenson and Slesnick (1983), and Pollak (1989). In practice, the Laspeyres price index is used to measure the Consumer Price Index (CPI) - see White (1999), Clements and Izan (1987). The Lapeyres formula does not take into account changes in the structure of consumption that occur as a result of price changes in a given time interval. It leads to the conclusion that the Laspeyres index can be biased due to the commodity substitution. Many economists consider the superlative indices (such as the Fisher index, the Walsh or the Törnqvist index) to be the best approximation of COLI (Von der Lippe 2007). Thus, any general classes of indices (such as the geo-logarithmic price index family) including these superlative index formulas seem to be especially interesting from the theoretical and practical point of view.

From a theoretical point of view, the feature of belonging to the geo-logarithmic price index family is a reason to consider the price index as good in the context of, for example, Martini's system of minimal requirements. The indices belonging to the discussed class have good properties, which is discussed in the further part of the article, although it cannot, of course, be said that an index outside this class does not have these properties. The author's modifications of the geo-logarithmic price index family proposed in the article yield indices that additionally fulfil the Laspeyres-Paasche bounding test, which is a desirable feature from the point of view of the economic approach. Moreover, it turns out that the relevant subclasses of one of the geo-logarithmic price index family modifications are notably close to the recognised superlative indices (the Fisher or Walsh indices), at the same time being their superset. From a practical point of view, the use of geo-logarithmic indices can also bring many benefits. If the world switches to the use of scanner data (e.g., in the CPI, HICP estimation, and so on) with "on-line" availability of data, then it could be possible to control $x$ and $y$ parameters (occurring in the class formula) to optimise that is, variance or mean square error in the geo-logarithmic index, used, for example, as the CPI (inflation) estimator. Thus, using, for example, a subclass where values fluctuate around superlative indices, it will be possible to select among those elements one that has 
distinctive statistical properties. Finally, the issue of geo-logarithmic indices seems to be interesting in itself, as there are still a few open, scientific problems. For example, one can inquire whether the range of index variability of this class is wider/narrower in relation to the variability range of superlative indices or whether some subclass of the geologarithmic class generates only superlative indices.

The article is organised as follows: Section 2 introduces the geo-logarithmic price index family, Section 3 presents its axiomatic properties and its particular subfamily, Section 4 provides generalisations of this family and discusses their properties and particular cases, Section 5 is a simulation study of all the considered index families, Section 6 is an empirical study, Section 7 provides some final comments and points out some open issues needing further research, Appendix (Section 8) contain definitions of basic index axioms and some computational details needed in the article.

\section{Geo-Logarithmic Price Index Family}

Let us consider a group of $N$ commodities observed at times $s, t$ (the time moment $s$ is considered as the basis) and let us denote:

$$
\begin{aligned}
p_{s} & =\left[p_{s 1}, p_{s 2}, \ldots, p_{s N}\right]^{\prime}-\text { a vector of prices at time } s \\
p_{t} & =\left[p_{t 1}, p_{t 2}, \ldots, p_{t N}\right]^{\prime}-\text { a vector of prices at time } t \\
q_{s} & =\left[q_{s 1}, q_{s 2}, \ldots, q_{s N}\right]^{\prime}-\text { a vector of quantities at time } s \\
q_{t} & =\left[q_{t 1}, q_{t 2}, \ldots, q_{t N}\right]^{\prime}-\text { a vector of quantities at time } t
\end{aligned}
$$

Let us denote by $\tau(x, y)$ the logarithmic mean of two positive real numbers $x$ and $y$, that is,

$$
\tau(x, y)=\frac{x-y}{\ln (x)-\ln (y)},
$$

if $x \neq y$ and $\tau(x, y)=x$ otherwise (Carlson 1972).

For $x, y \in[0,1]$, let $q^{x}$ and $q^{y}$ be two vectors whose components are defined as follows

$$
q_{i}^{x}=q_{t i}^{x} q_{s i}^{1-x}, \quad q_{i}^{y}=q_{t i}^{y} q_{s i}^{1-y}, \quad \text { for } \quad i=1,2, \ldots, N
$$

and let

$$
\begin{aligned}
& w_{t i}^{x}=\frac{p_{t i} q_{i}^{x}}{\sum_{i=1}^{N} p_{t i} q_{i}^{x}}, \\
& w_{s i}^{y}=\frac{p_{s i} q_{i}^{y}}{\sum_{i=1}^{N} p_{s i} q_{i}^{y}} .
\end{aligned}
$$

The geo-logarithmic, or the $P_{x y}$, family is the class of price indices defined by (Fattore 2006)

$$
P_{x y}\left(q_{s}, q_{t}, p_{s}, p_{t}\right)=\prod_{i=1}^{N}\left(\frac{p_{t i}}{p_{s i}}\right)^{\nu_{i}^{x y}}
$$


where weights $v_{i}^{x y}$ are as follows

$$
\nu_{i}^{x y}=\frac{\tau\left(w_{t i}^{x}, w_{s i}^{y}\right)}{\sum_{j=1}^{N} \tau\left(w_{t j}^{x}, w_{s j}^{y}\right)} .
$$

The following theorem (Fattore 2010) is the fundamental result for the $P_{x y}$ parameterisation.

Theorem 1. The mapping associating the pair $(x, y) \in[0,1] \times[0,1]$ with the index $P_{x y}$ is one to one, that is, if $(x, y) \neq(u, v)$, then $P_{x y} \neq P_{u v}$.

\section{Axiomatic Properties of Geo-Logarithmic Price Indices}

The geo-logarithmic family of price indices was proposed by the Italian statistician Martini (1992a). As was mentioned before, from a theoretical point of view, a wellconstructed index should satisfy a group of postulates (tests) arising from the axiomatic index theory. Although there is no universal agreement on the axiomatic properties for a formula to be considered as an index (IMF 2004), one of commonly accepted systems of minimum requirements for the price index formula comes also from Martini (1992b). Obviously, each $P_{x y}$ index satisfies identity and since Theorem 2 holds (Subsection 3.1), the geo-logarithmic price indices fulfil Martini's minimal requirements.

\subsection{List of Axioms}

In Fattore (2010), we can find proof of the following theorems.

Theorem 2. Geo-logarithmic price indices $P_{x y}$ satisfy: (1) proportionality, (2) commensurability and (3) homogeneity. Moreover, the basis reversibility axiom holds if and only if $y=1-x$.

Theorem 3. An index from the $P_{x y}$ class is monotonic if and only if $x=y$.

The immediate conclusion from Theorem 2 and Theorem 3 is the fact that the only monotonic geo-logarithmic price index being basis reversible is $P_{0.5} 0.5$ (Subsection 3.2). In Fattore (2010), it is proved that the only factor reversible element of the $P_{x y}$ family is the Sato-Vartia index $P_{10}$ (Von der Lippe 2007).

\subsection{Special Subfamily $P_{x x}$}

Since Theorem 3 holds and taking into consideration the monotonicity axiom from the EVsystem, it seems interesting to consider a special subfamily $P_{x x}$. Let us note that for $x=y$ from (5) and (6) we obtain (Fattore 2010)

$$
P_{x x}=\frac{\sum_{i=1}^{N} p_{t i} q_{i}^{x}}{\sum_{i=1}^{N} p_{s i} q_{i}^{x}}=\frac{\sum_{i=1}^{N} p_{t i} q_{t i}^{x} q_{s i}^{1-x}}{\sum_{i=1}^{N} p_{s i} q_{t i}^{x} q_{s i}^{1-x}} .
$$

In particular, we obtain some known price index formulas. For instance, the Laspeyres $\left(P_{\mathrm{La}}\right)$, Paasche $\left(P_{\mathrm{Pa}}\right)$ and Walsh $\left(P_{\mathrm{W}}\right)$ price indices can be expressed as 


$$
\begin{gathered}
P_{\mathrm{La}}=\frac{\sum_{i=1}^{N} p_{t i} q_{s i}}{\sum_{i=1}^{N} p_{s i} q_{s i}}=P_{00}, \\
P_{\mathrm{Pa}}=\frac{\sum_{i=1}^{N} p_{t i} q_{t i}}{\sum_{i=1}^{N} p_{s i} q_{t i}}=P_{11}, \\
P_{\mathrm{W}}=\frac{\sum_{i=1}^{N} p_{t i} \sqrt{q_{s i} q_{t i}}}{\sum_{i=1}^{N} p_{s i} \sqrt{q_{s i} q_{t i}}}=P_{0.5} \quad 0.5 .
\end{gathered}
$$

\section{Example 1}

Let us take into consideration a group of $N=12$ commodities, where prices and quantities at time moments $s$ and $t$ are presented in Table 1. Figure 1 presents functions $P_{x y}$ and $P_{x x}$ for

Table 1. The values of prices and quantities at time moments $s$ and $t$.

\begin{tabular}{|l|r|r|r|c|}
\hline Commodity & $q_{s}$ & $q_{t}$ & $p_{s}$ & $p_{t}$ \\
\hline 1 & 350 & 200 & 900 & 1000 \\
\hline 2 & 550 & 200 & 1600 & 1700 \\
\hline 3 & 5000 & 3000 & 460 & 500 \\
\hline 4 & 710 & 500 & 3 & 3.2 \\
\hline 5 & 350 & 340 & 100 & 105 \\
\hline 6 & 890 & 700 & 1000 & 1150 \\
\hline 7 & 850 & 800 & 900 & 1000 \\
\hline 8 & 600 & 500 & 1530 & 1600 \\
\hline 9 & 5000 & 3000 & 480 & 500 \\
\hline 10 & 700 & 500 & 4 & 4.2 \\
\hline 11 & 550 & 340 & 100 & 110 \\
\hline 12 & 800 & 700 & 1000 & 1100 \\
\hline
\end{tabular}
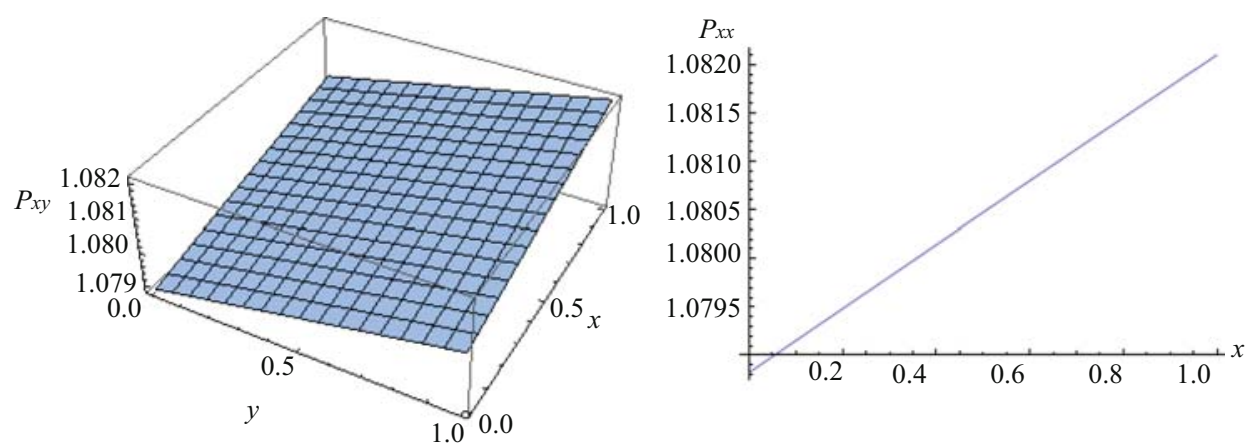

Fig. 1. Functions $P_{x y}$ and $P_{x x}$ depending on $x$ and $y$ for dataset described in Table 1. 

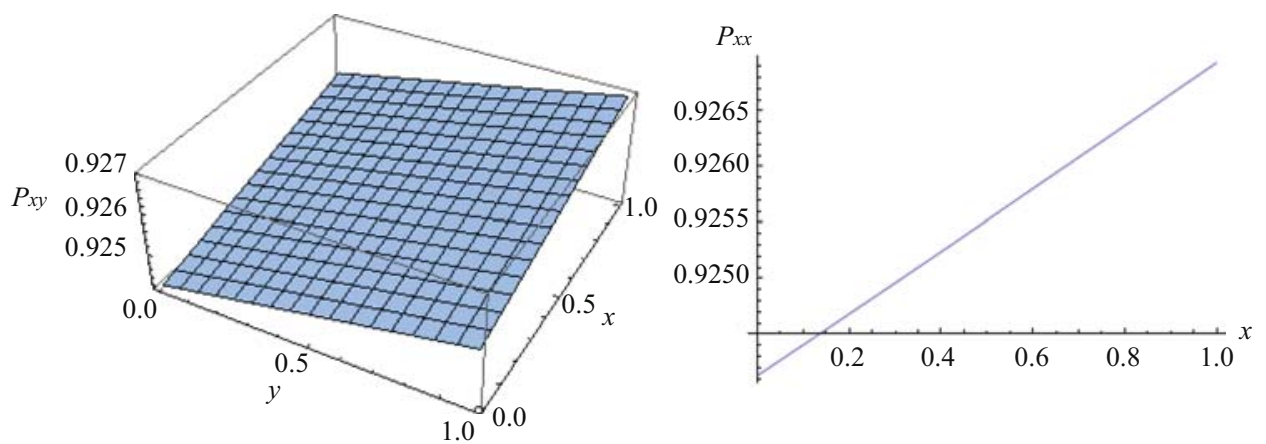

Fig. 2. Functions $P_{x y}$ and $P_{x x}$ depending on $x$ and $y$ for the reverse case ( $t$ is the base period).

$x, y \in[0,1]$. Figure 2 presents functions $P_{x y}$ and $P_{x x}$ for the reverse case, that is, when the moment $t$ is treated as the base period. It suggests that in the case of negative correlation between prices and quantities, the $P_{x y}$ formula is a monotonic (here increasing) function of its arguments, that is, in our example the value of $P_{x y}$ goes up if $x$ or $y$ increases. If the suggestion were true, from (8) and (9) we would have an immediate conclusion that $P_{x y}$ satisfies the Laspeyres-Paasche bounding test. In fact, it is not generally true (see Subsection 3.3).

\subsection{Geo-logarithmic Price Indices and the Laspeyres-Paasche Bounding Test}

The Consumer Price Index approximates changes in costs of household consumption assuming constant utility, particularly in settings where COLI, Cost of Living Index, is chosen as a target for the index. In the so-called economic approach, the upper and lower bounds for the COLI are provided by the Laspeyres and Paasche price index formulas. If the price index value is within these bounds, then we say that this price index satisfies the Laspeyres-Paasche bounding test belonging to the group of mean value tests (Von der Lippe 2007).

\section{Example 2}

Let us take into consideration a group of $N=4$ commodities where prices and quantities at time moments $s$ and $t$ are presented in Table 2. Figure 3 presents the function $P_{x y}$ for $x, y \in[0,1]$. Figure 4 presents the function $P_{x 1}$ for $x \in[0,1]$. Figure 5 presents the function $P_{x x}$ for $x \in[0,1]$.

Table 2. The values of prices and quantities at time moments $s$ and $t$.

\begin{tabular}{|l|c|r|r|r|}
\hline Commodity & $q_{s}$ & $q_{t}$ & $p_{s}$ & \multicolumn{1}{c|}{$p_{t}$} \\
\hline 1 & 300 & 200 & 80 & 90 \\
\hline 2 & 1200 & 900 & 500 & 550 \\
\hline 3 & 2000 & 1 & 120 & 130 \\
\hline 4 & 4.1 & 4 & 30000 & 31500 \\
\hline
\end{tabular}




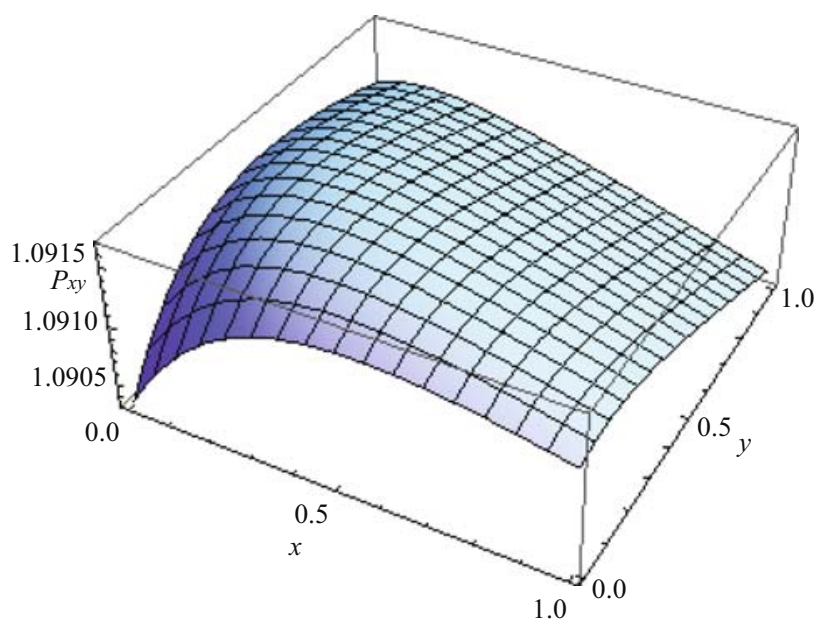

Fig. 3. Function $P_{x y}$ depending on $x$ and $y$ for dataset described in Table 2.

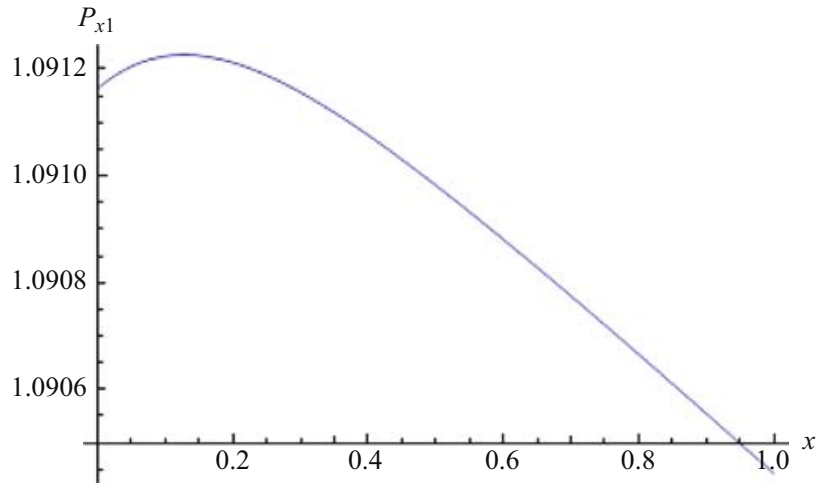

Fig. 4. Function $P_{x 1}$ depending on $x$ for dataset described in Table 2.

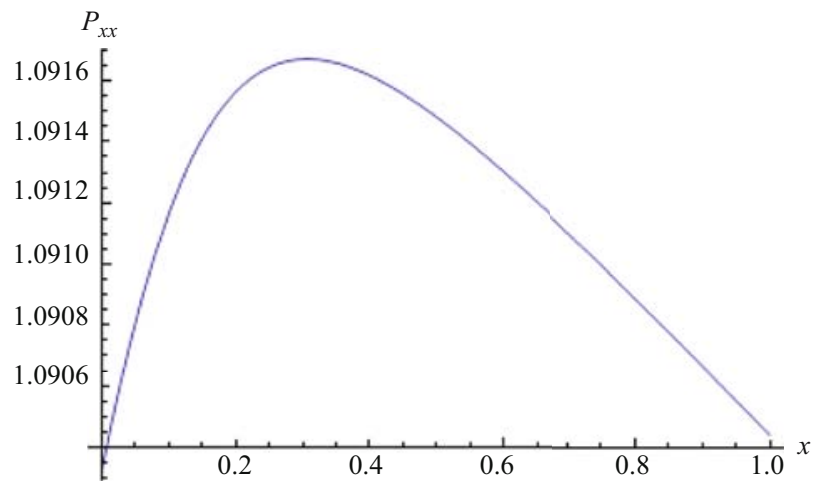

Fig. 5. Function $P_{x x}$ depending on $x$ for dataset described in Table 2. 
Observing Figures 1, 2 and 3, we conclude that even if changes between prices and quantities are inversely related, the indices from $P_{x y}$ or $P_{x x}$ families may fail the Laspeyres-Paasche bounding test since $P_{00}=P_{\mathrm{La}}$ and $P_{11}=P_{\mathrm{Pa}}$. Moreover, the $P_{x y}$ formula does not have to be a monotonic function of its arguments. Obviously, the quantity response to price changes is extremely strong in the case of commodity number 3 and it would not be observed in practice. Nevertheless, any considered and accepted test from the axiomatic price index theory must hold for any vectors of prices and quantities. The following question arises: what about the case when the quantity response is not so extreme (it is naturally limited) and still prices and quantities are inversely related? To answer this question, we run a simulation study (see Section 5) in which the parameter connected with the quantity changes is controlled.

\subsection{Geo-logarithmic Price Indices and Superlative Indices}

Following the economic approach to the price index theory, Diewert proposed the special family of indices that he called superlative (Diewert 1976). Although the axiomatic and the economic approaches differ from each other, connections between them are worth studying (Von der Lippe 2007). Fattore (2010) has proven that the only superlative index number belonging to the geo-logarithmic family is the Walsh index $\left(P_{0.5} 0.5\right)$. Among superlative price indices, a very important role is played by the Törnqvist index:

$$
P_{\mathrm{T}}=\prod_{i=1}^{N}\left(\frac{p_{t i}}{p_{s i}}\right)^{\frac{w_{s i}^{0}+w_{t i}^{1}}{2}},
$$

which does not belong to the $P_{x x}$ family (Fattore 2010). Nevertheless, in the same paper it is proved that the first-order approximation of the geo-logarithmic price index has a Törnqvist-like form. Similarly, the Fisher price index

$$
P_{\mathrm{F}}=\sqrt{P_{\mathrm{La}} P_{\mathrm{Pa}}}
$$

is not a member of the geo-logarithmic price index family but since the superlative Fisher and Törnqvist indices approximate each other (Dumagan 2002), the Fisher price index also should approximate the geo-logarithmic price indices.

\section{Example 3}

Let us use data from Example 1. Figure 6 presents the function $\left|P_{x y}-P_{\mathrm{F}}\right|$ depending on $x, y \in[0,1]$. Figure 7 presents the function $\left|P_{x x}-P_{\mathrm{F}}\right|$ depending on $x \in[0,1]$.

We observe (See Figure 6) that the best Fisher index approximation that uses $P_{x y}$ indices is obtained here for $y=1-x$. The $P_{x 1-x}$ subfamily was investigated by Fattore (2010). He has proven that $P_{x 1-x}$ indices satisfy the Martini's minimal requirements.

Let us also note that the basis reversibility axiom holds if and only if $y=1-x$ (see Theorem 2). Thus, further investigations on the $P_{x 1-x}$ subfamily seem to be especially interesting. Observing Figure 7, we can see that the best Fisher index approximation that uses $P_{x x}$ formulas is obtained for $x=0.5$, which leads to the Walsh price index $\left(P_{0.5} 0.5\right)$ being the only monotonic element of the $P_{x 1-x}$ subfamily. It is not surprising since the superlative price indices approximate each other. However, this is not the best Fisher price 


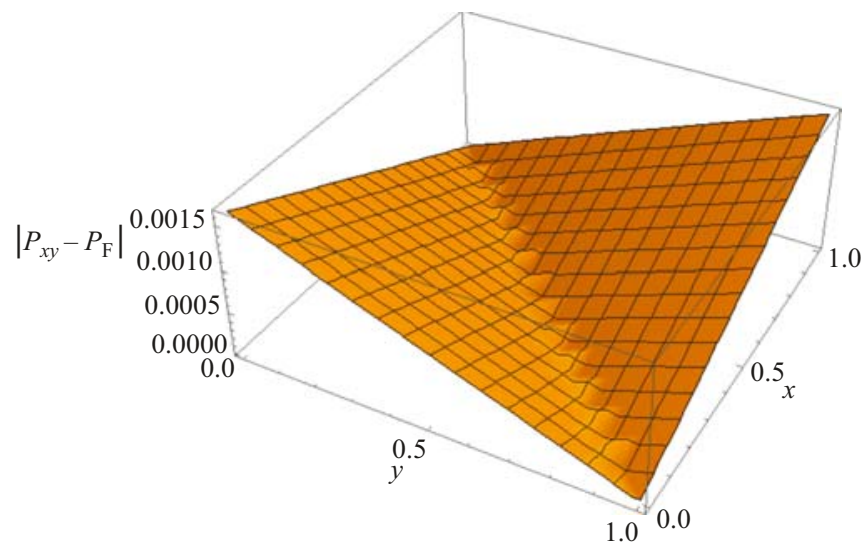

Fig. 6. Function $\left|P_{x y}-P_{F}\right|$ depending on $x, y \in[0,1]$ for dataset described in Table 1 .

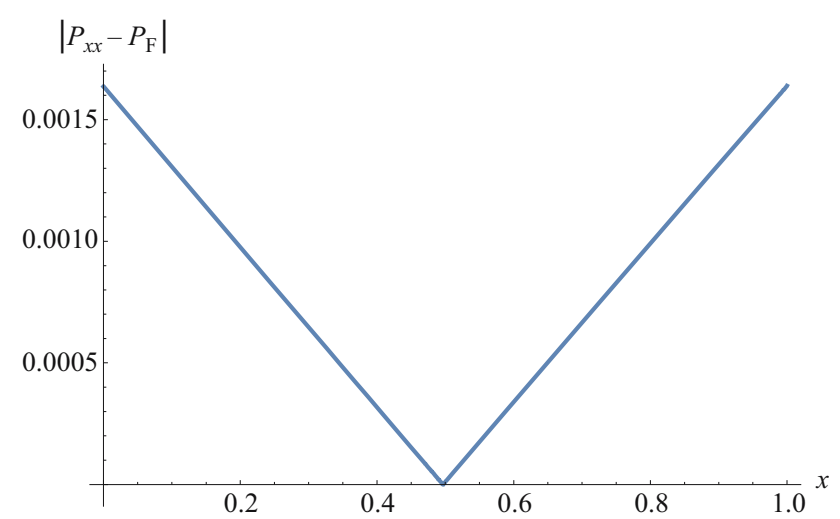

Fig. 7. Function $\left|P_{x x}-P_{\mathrm{F}}\right|$ depending on $x \in[0,1]$ for dataset described in Table 1.

index approximation in our study, that is, although $P_{\mathrm{W}}=1.08047 \approx P_{\mathrm{F}}=1.08046$, the index $P_{01}$ seems to be a better proxy for the Fisher index value (See Figure 8 ). Please note that the $P_{01}$ index is not the Sato-Vartia price index (it is easy to verify that, in general, values of $P_{01}$ differ from values of $P_{10}$ ).

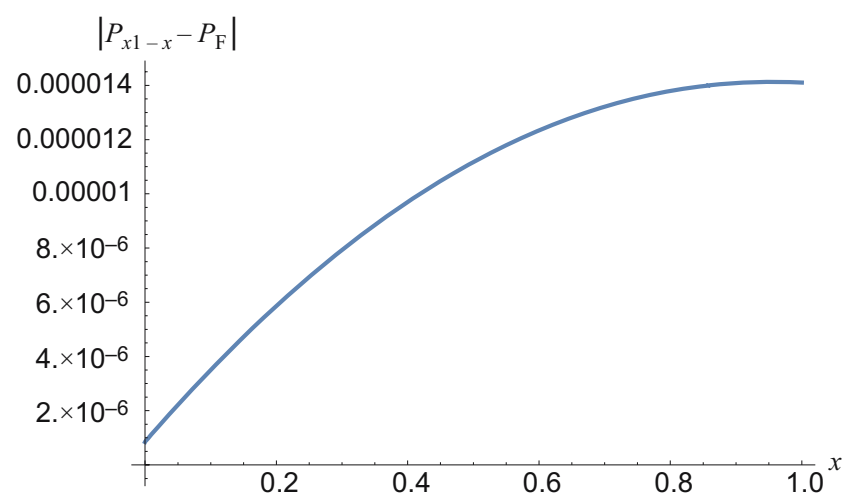

Fig. 8. Function $\left|P_{x 1-x}-P_{\mathrm{F}}\right|$ depending on $x \in[0,1]$ for dataset described in Table 1 . 


\section{Generalisation of the Geo-Logarithmic Price Index Family}

We consider two problems here. Firstly, it would be interesting to modify the structure of the geo-logarithmic family to obtain the price index family $\tilde{P}_{x y z}$ including the Fisher index. Secondly, we intend to verify consequences of changing the weighted geometric mean into the weighted arithmetic mean of quantities in $\tilde{P}_{x x z}$ subfamily.

\subsection{Generalisation Through an Additional Parameter}

Similarly to (2), (3), (4) and (6), let us denote by

$$
\begin{gathered}
q_{i}^{A x}=q_{t i}^{x} q_{s i}^{1-x}, \quad q_{i}^{A y}=q_{t i}^{y} q_{s i}^{1-y}, \\
q_{i}^{B x}=q_{t i}^{1-x} q_{s i}^{x}, \quad q_{i}^{B y}=q_{t i}^{1-y} q_{s i}^{y}, \\
w_{t i}^{A x}=\frac{p_{t i} q_{i}^{A x}}{\sum_{i=1}^{N} p_{t i} q_{i}^{A x}}, \quad w_{s i}^{A y}=\frac{p_{s i} q_{i}^{A y}}{\sum_{i=1}^{N} p_{s i} q_{i}^{A y}}, \\
w_{t i}^{B x}=\frac{p_{t i} q_{i}^{B x}}{\sum_{i=1}^{N} p_{t i} q_{i}^{B x}}, \quad w_{s i}^{B y}=\frac{p_{s i} q_{i}^{B y}}{\sum_{i=1}^{N} p_{s i} q_{i}^{B y}}, \\
v_{A i}^{x y}=\frac{\tau\left(w_{t i}^{A x}, w_{s i}^{A y}\right)}{\sum_{j=1}^{N} \tau_{\left(w_{t j}^{A x}, w_{s j}^{A y}\right)}}, \quad v_{B i}^{x y}=\frac{\tau\left(w_{t i}^{B x}, w_{s i}^{B y}\right)}{\sum_{j=1}^{N} \tau\left(w_{t j}^{B x}, w_{s j}^{B y}\right)},
\end{gathered}
$$

for $i=1,2, \ldots, N, x, y \in[0,1]$.

Under significations (13)-(17), we define the new class of price indices $\left(\tilde{P}_{x y z}\right)$ as follows

$$
\tilde{P}_{x y z}=\left\{\prod_{i=1}^{N}\left(\frac{p_{t i}}{p_{s i}}\right)^{v_{A i}^{x y}}\right\}^{z}\left\{\prod_{i=1}^{N}\left(\frac{p_{t i}}{p_{s i}}\right)^{v_{B i}^{x y}}\right\}^{1-z}, \quad \text { for } x, y, z \in[0,1] .
$$

Firstly, let us note that for fixed values of $x, y$ and $z$ the price index $\tilde{P}_{x y z}$ fulfils the Martini's minimal requirements since it can be expressed as a weighted geometric mean of two price indices (with weights $z$ and $1-z$ ), satisfying the Martini's minimal requirements (see Appendix B, Subsection 8.2). In fact, these two price indices (defined inside curly brackets in Equation 18) satisfy the Martini's minimal requirements. The first one (on the left side of Equation 18) is identical with $P_{x y}$ index (for fixed values of $x$ and $y$ ) and its axiomatic properties were proved by Fattore (2010). The proof of the same group of axioms in the case of the second price index (inside curly brackets on the right side of Equation18) would be analogous. 
Secondly, let us note that the following relation holds

$$
\tilde{P}_{x y 1}=\tilde{P}_{1-x \quad 1-y} 0=P_{x y}, \quad \text { for } \quad x, y \in[0,1],
$$

which means that the $P_{x y}$ family is a special case of the $\tilde{P}_{x y z}$ family.

Moreover, $\tilde{P}_{101}$ and $\tilde{P}_{010}$ are the Sato-Vartia indices and also we obtain

$$
\begin{gathered}
\tilde{P}_{001}=\tilde{P}_{110}=P_{\mathrm{La}}, \\
\tilde{P}_{111}=\tilde{P}_{000}=P_{\mathrm{Pa}}, \\
\tilde{P}_{\frac{111}{222}}=P_{\mathrm{W}}
\end{gathered}
$$

and, what is more interesting, we have

$$
\tilde{P}_{00 \frac{1}{2}}=\tilde{P}_{11 \frac{1}{2}}=P_{\mathrm{F}}
$$

Finally, the following approximation can be proved (see Appendix C, Subsection 8.3).

$$
\forall i \in\{1,2, \ldots, N\} \quad q_{s i} \approx q_{t i} \wedge w_{s i}^{x} \approx w_{t i}^{y} \Rightarrow \tilde{P}_{x y z} \approx P_{\mathrm{T}} .
$$

\section{Example 4}

Let us use data from Example 1. Figure 9 presents the function $\tilde{P}_{x y \frac{1}{2}}$ depending on $x, y \in[0,1]$.

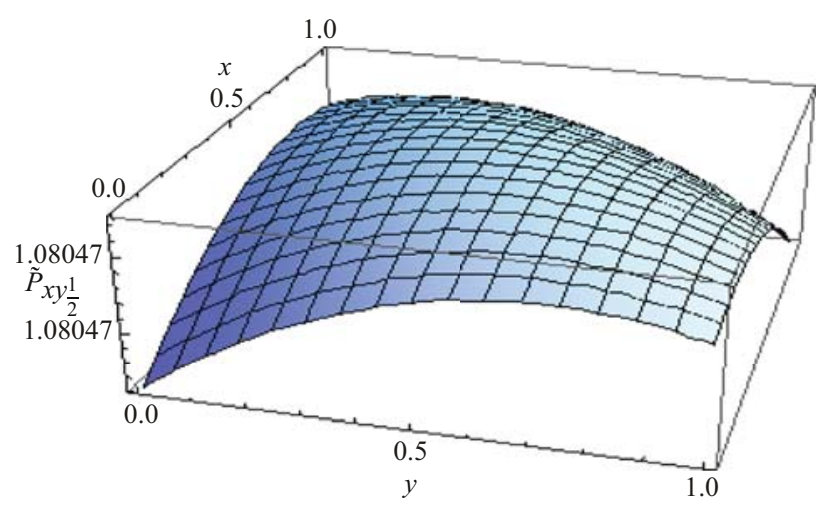

Fig. 9. Function $\tilde{P}_{x y \frac{1}{2}}$ depending on $x, y \in[0,1]$ for dataset described in Table 1.

As we can see, the interval of values of indices from the considered family (for $z=0.5)$ is very narrow and they fluctuate around superlative index values $\left(P_{\mathrm{W}}=1.08047, P_{\mathrm{F}}=1.08046\right)$.

\section{Example 5}

Let us take into consideration a group of $N=5$ commodities where prices and quantities at time moments $s$ and $t$ are presented in Table 3. Figure 10 presents the function $P_{x y}$ for $x, y \in[0,1]$. Figure 11 presents the function $\tilde{P}_{x y \frac{1}{2}}$ for $x, y \in[0,1]$. Similarly to the results 
obtained in the Example 4, the interval of values of indices from the $\tilde{P}_{x y \frac{1}{2}}$ family is very narrow. We compare its range with the range obtained for a class of superlative price indices introduced by Diewert (1976). The Diewert's proposition of the above-mentioned class of indices is as follows

$$
P_{\mathrm{D}}(r)=\left(\frac{\sum_{i=1}^{N}\left(\frac{p_{t i}}{p_{s i}}\right)^{\frac{r}{2}} \frac{p_{s i} q_{s i}}{p_{s} q_{s}}}{\sum_{i=1}^{N}\left(\frac{p_{s i}}{p_{t i}}\right)^{\frac{r}{2}} \frac{p_{t i} q_{t i}}{p_{t} q_{t}}}\right)^{\frac{1}{r}},
$$

Table 3. The values of prices and quantities at time moments $s$ and $t$.

\begin{tabular}{|l|r|r|r|r|}
\hline Commodity & $q_{s}$ & $q_{t}$ & $p_{s}$ & $p_{t}$ \\
\hline 1 & 100 & 70 & 80 & 90 \\
\hline 2 & 820 & 900 & 500 & 550 \\
\hline 3 & 20000 & 15000 & 120 & 130 \\
\hline 4 & 50 & 40 & 30000 & 31500 \\
\hline 5 & 4000 & 3000 & 3 & 3.5 \\
\hline
\end{tabular}

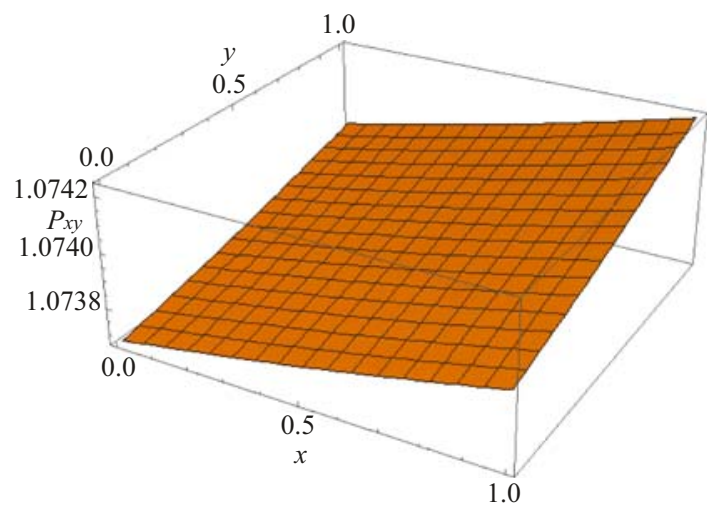

Fig. 10. Function $P_{x y}$ depending on $x$ and $y$ for dataset described in Table 3.

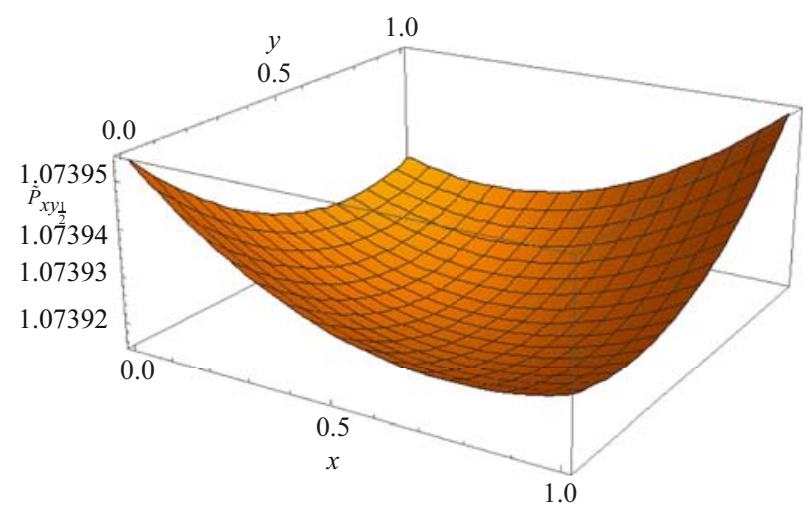

Fig. 11. Function $\tilde{P}_{x y \frac{1}{2}}$ depending on $x$ and $y$ for dataset described in Table 3. 
where $r \in R \backslash\{0\}$ and

$$
p_{\tau} q_{\tau}=\sum_{k=1}^{N} p_{\tau k} q_{\tau k}, \quad \text { for } \quad \tau=s, t
$$

Figure 12 presents the function $P_{\mathrm{D}}(r)$ for $r \in[-1000,1000] \backslash\{0\}$. After optimisation of functions $P_{x y}, \tilde{P}_{x y \frac{1}{2}}$ and $P_{\mathrm{D}}(r)$ we obtain their following ranges: $P_{x y} \in[1.07367,1.07424]$, $\tilde{P}_{x y \frac{1}{2}} \in[1.07392,1.07395]$ and $P_{\mathrm{D}}(r) \in[1.07393,1.10587]$. The length of the interval of possible index values is the smallest in the case of the family $\tilde{P}_{x y \frac{1}{2}}$. The open question is whether the above conclusion has a general character.

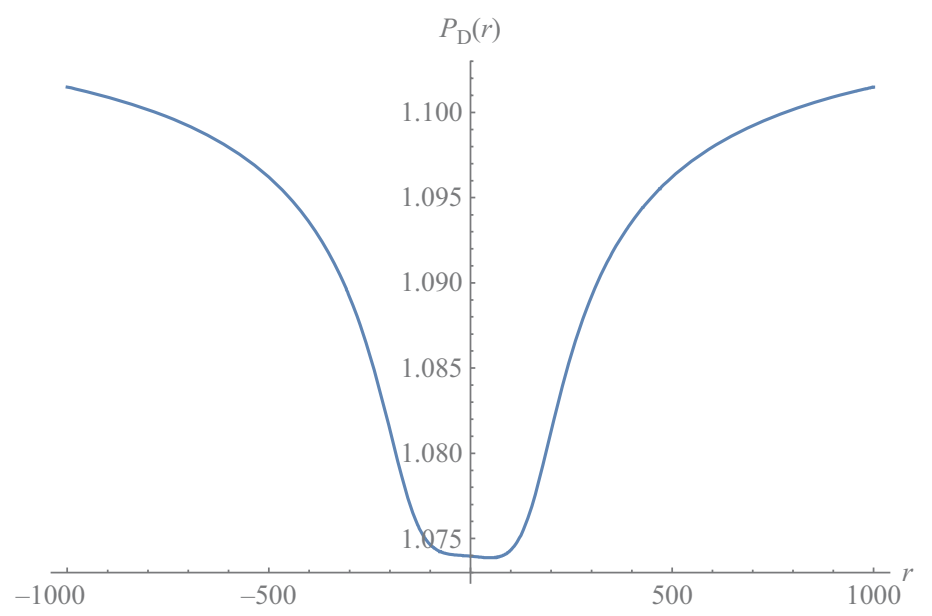

Fig. 12. Function $P_{\mathrm{D}}(r)$ for $r \in[-1000,1000] \backslash\{0\}$.

\subsection{Modification Through Mean Change}

Fattore (2010) shows that

$$
\prod_{i=1}^{N}\left(\frac{p_{t i}}{p_{s i}}\right)^{v_{A i}^{k x}}=\frac{\sum_{i=1}^{N} p_{t i} q_{t i}^{x} q_{s i}^{1-x}}{\sum_{i=1}^{N} p_{s i} q_{t i}^{x} q_{s i}^{1-x}}
$$

and, by the analogy, we obtain

$$
\prod_{i=1}^{N}\left(\frac{p_{t i}}{p_{s i}}\right)^{v_{B i}^{r x}}=\frac{\sum_{i=1}^{N} p_{t i} q_{t i}^{1-x} q_{s i}^{x}}{\sum_{i=1}^{N} p_{s i} q_{t i}^{1-x} q_{s i}^{x}}
$$

From (18), (27) and (28) we obtain

$$
\tilde{P}_{x x z}=\left(\frac{\sum_{i=1}^{N} p_{t i} q_{t i}^{x} q_{s i}^{1-x}}{\sum_{i=1}^{N} p_{s i} q_{t i}^{x} q_{s i}^{1-x}}\right)^{z}\left(\frac{\sum_{i=1}^{N} p_{t i} q_{t i}^{1-x} q_{s i}^{x}}{\sum_{i=1}^{N} p_{s i} q_{t i}^{1-x} q_{s i}^{x}}\right)^{1-z}
$$


Let us note that if we change the geometric mean of quantities into the arithmetic mean of quantities in the $\tilde{P}_{x x z}$ formula, we obtain

$$
\tilde{P}_{x x z}^{A}=\left(\frac{\sum_{i=1}^{N} p_{t i}\left(x q_{t i}+(1-x) q_{s i}\right.}{\sum_{i=1}^{N} p_{s i}\left(x q_{t i}+(1-x) q_{s i}\right.}\right)^{z}\left(\frac{\sum_{i=1}^{N} p_{t i}\left((1-x) q_{t i}+x q_{s i}\right)}{\sum_{i=1}^{N} p_{s i}\left((1-x) q_{t i}+x q_{s i}\right)}\right)^{1-z}
$$

This is still a quite general family of indices. In particular, we have

$$
\begin{gathered}
\tilde{P}_{00 \frac{1}{2}}^{A}=\tilde{P}_{11 \frac{1}{2}}^{A}=P_{\mathrm{F}}, \\
\tilde{P}_{000}^{A}=\tilde{P}_{111}^{A}=P_{\mathrm{Pa}}, \\
\tilde{P}_{001}^{A}=\tilde{P}_{110}^{A}=P_{\mathrm{La}}, \\
\tilde{P}_{\frac{11 \frac{1}{2}}{2}}^{A}=P_{\mathrm{ME}} .
\end{gathered}
$$

where $P_{\mathrm{ME}}$ denotes the Marshal-Edgeworth price index (see Von der Lippe 2007).

What is more interesting, the following theorem can be proved (see Appendix D, Subsection 8.4).

Theorem 4. Each price index from the $\tilde{P}_{x x z}^{A}$ subfamily satisfies the Laspeyres-Paasche bounding test.

\subsection{Properties of Cofactors of Modified Geo-logarithmic Price Indices}

"Index numbers come in pairs in economic theory, one of price and the other a matching one of quantity. In economic practice they tend to be found paired off in this way (. . .). Such a pair may be designed to account for the variation in a value aggregate, as when movements in aggregate expenditure of consumers are analysed into the two components of changes in prices and in real consumption" (Allen 1975, 1).

According to the cited fragment and to ensure the joint consistency of both price and quantity comparisons it could be desirable in practice using such price indices which, together with their cofactors, satisfy fundamental tests from axiomatic index theory.

Let us note that for the given sets of prices and quantities, described by $N$-dimensional vectors $p_{s}, p_{t}, q_{s}$ and $q_{t}$ (see Section 2 ), the ratio

$$
V\left(q_{s}, q_{t}, p_{s}, p_{t}\right)=\frac{\sum_{i=1}^{N} p_{t i} q_{t i}}{\sum_{i=1}^{N} p_{s i} q_{s i}}
$$

is called the value index between time moments $s$ and $t$. The aim of the price and quantity index theory is to decompose the value index as the product of two strictly positive functions

$$
V\left(q_{s}, q_{t}, p_{s}, p_{t}\right)=P\left(q_{s}, q_{t}, p_{s}, p_{t}\right) \cdot Q\left(q_{s}, q_{t}, p_{s}, p_{t}\right)
$$


where $P$ and $Q$ denote the well-defined price and quantity indices. The given price index formula $P\left(q_{s}, q_{t}, p_{s}, p_{t}\right)$ has its associated cofactor defined by

$$
\operatorname{cof} P\left(q_{s}, q_{t}, p_{s}, p_{t}\right)=\frac{V\left(q_{s}, q_{t}, p_{s}, p_{t}\right)}{P\left(q_{s}, q_{t}, p_{s}, p_{t}\right)} .
$$

From (36) and (37) we have that the cofactor of a given price index is the associated quantity index. The geo-logarithmic price index family has the distinctive feature that the cofactors of its elements satisfy the proportionality and homogeneity axioms (see Appendix A, Subsection 8.1). From the axiomatic index theory (Balk 1995), we know that only the fulfilment of the factor reversibility axiom guarantees that the cofactor (with respect to quantities) satisfies all properties fulfilled by price index itself (with respect to prices). It can be easily explained since in that case the cofactor and the price index share the same functional form (Fattore 2010). As it is known, the factor reversibility test is very restrictive and it rules out most indices commonly used in practice, such as the Laspeyres index (Von der Lippe 2007). Many authors treat this axiom as a nonessential property. To ensure the joint consistency of both price and quantity comparisons, alternatively we can search for a class of price indices satisfying at least an important subset of fundamental axioms together with their cofactors. In this sense, such a class of indices can be considered "good". Motivated by looking for such a "good class", Martini (1992) proposed the geo-logarithmic price index family.

In the paper by Fattore (2010), it is proved that cofactors of geo-logarithmic price indices satisfy the proportionality and homogeneity axioms (see Proposition 10 and its proof in this original work). Since the proportionality holds for any $x, y \in[0,1]$ and for any positive real number $k$, we have

$$
\operatorname{cof} P_{x y}\left(q_{s}, q_{t}, p_{s}, k p_{s}\right)=k .
$$

From (19) and (38) we conclude that

$$
\operatorname{cof} \tilde{P}_{x y 1}\left(q_{s}, q_{t}, p_{s}, k p_{s}\right)=k .
$$

Since the equality (39) holds for any $x, y \in[0,1]$, we obtain as a consequence

$$
\operatorname{cof} \tilde{P}_{1-x} 1-y \quad{ }_{1}\left(q_{s}, q_{t}, p_{s}, k p_{s}\right)=k
$$

Let us note that any index from the $\tilde{P}_{x y z}$ family can be written as

$$
\tilde{P}_{x y z}=\left(\tilde{P}_{x y 1}\right)^{z}\left(\tilde{P}_{1-x} 1-y \quad 1\right)^{1-z} .
$$

From (41) we have

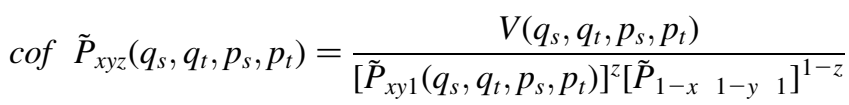

$$
\begin{aligned}
& =\left[\frac{V\left(q_{s}, q_{t}, p_{s}, p_{t}\right)}{\tilde{P}_{x y 1}\left(q_{s}, q_{t}, p_{s}, p_{t}\right)}\right]^{z}\left[\frac{V\left(q_{s}, q_{t}, p_{s}, p_{t}\right)}{\tilde{P}_{1-x} 1-y \quad 1\left(q_{s}, q_{t}, p_{s}, p_{t}\right)}\right]^{1-z},
\end{aligned}
$$


and it leads to the following conclusion

$$
\begin{aligned}
\operatorname{cof} \tilde{P}_{x y z}\left(q_{s}, q_{t}, p_{s}, p_{t}\right)= & {\left[\operatorname{cof} \tilde{P}_{x y 1}\left(q_{s}, q_{t}, p_{s}, p_{t}\right)\right]^{z} } \\
& \cdot\left[\begin{array}{llll}
\operatorname{cof} & \tilde{P}_{1-x} & 1-y & 1
\end{array}\left(q_{s}, q_{t}, p_{s}, p_{t}\right)\right]^{1-z}
\end{aligned}
$$

From (39), (40) and (43) we obtain

$$
\text { cof } \tilde{P}_{x y z}\left(q_{s}, q_{t}, p_{s}, k p_{s}\right)=k^{z} k^{1-z}=k \text {. }
$$

Thus, cofactors of $\tilde{P}_{x y z}$ indices satisfy the proportionality axiom. The proof for the homogeneity could be done analogically. Let us note that the problem with these axioms appears in the case of the $\tilde{P}_{x x z}^{A}$ index family because weighting by arithmetic means of quantities makes the cofactors violating the proportionality axiom. In our opinion, it does not mean that such a choice of weights is wrong and cannot be accepted since indices from the $\tilde{P}_{x x z}^{A}$ family satisfy Martini's minimal requirements and they fulfil the LaspeyresPaasche bounding test. Moreover, these indices remain quite stable even when prices are strongly fluctuated (see Simulation 2 in Section 5).

\section{Simulation Study}

\section{Simulation 1}

Let us take into consideration a group of $N=12$ components where prices and quantities are normally distributed as follows:

$$
\begin{aligned}
& p_{i}^{\tau} \sim N\left(p_{i 0}^{\tau}, v_{i}^{\tau} p_{i 0}^{\tau}\right) \\
& q_{i}^{\tau} \sim N\left(q_{i 0}^{\tau}, u_{i}^{\tau} q_{i 0}^{\tau}\right)
\end{aligned}
$$

where $\tau=s, t, N(\mu, \sigma)$ denotes the normal distribution with the mean $\mu$ and the standard deviation $\sigma, v_{i}^{\tau}$ denotes the volatility coefficient (coefficient of variation) of the $i-$ th price at time $\tau$, i.e., $v_{i}^{\tau}=D\left(p_{i}^{\tau}\right) / p_{i 0}^{\tau}, u_{i}^{\tau}$ denotes the volatility coefficient of the $i-$ th quantity at time $\tau$, i.e., $u_{i}^{\tau}=D\left(q_{i}^{\tau}\right) / q_{i 0}^{\tau}$. Before generating prices and quantities, we generated values of volatility coefficients using uniform distributions, that is, $v_{i}^{\tau} \sim$ $U\left(0, v^{\tau}\right)$ and $u_{i}^{\tau} \sim U\left(0, u^{\tau}\right)$. Expected values of prices and quantities are described by vectors from Example 1, that is,

$$
\begin{aligned}
& P_{0}^{t}=[1000,1700,500,3.2,105,1150,1000,1600,500,4.2,110,1100]^{\prime} ; \\
& P_{0}^{s}=[900,1600,460,3,100,1000,900,1530,480,4,100,1000]^{\prime} ; \\
& Q_{0}^{t}=[200,200,3000,500,340,700,800,500,3000,500,340,700]^{\prime} ; \\
& Q_{0}^{s}=[350,550,5000,710,350,890,850,600,5000,700,550,800]^{\prime} .
\end{aligned}
$$

In our experiment, we are going to control values of volatility coefficients of prices and quantities by setting values of $v^{s}, v^{t}, u^{s}, u^{t}$ and observe their influence on the discussed 
general indices and their distance to the Laspeyres and Paasche formulas. We consider several cases, that is, Case 1 (the volatilities of price and quantity processes are low and the quantity response to price changes is quite normal - see Example 1), Case 2 (the volatilities of prices and quantities are large, the quantity response to price changes is strongly fluctuated), Case 3 (the volatility of prices is small but the volatility of quantities is large, that is, the quantity response to price changes may be strong), Case 4 (the volatility of prices is large but the volatility of quantities is small, that is, the quantity response to price changes is rather small). For each case, we generate values of price and quantity vectors in $n=1000$ repetitions. Let us denote for fixed values of $x$ and $y$ and for each of $k$ th repetition:

$$
\begin{aligned}
& \Delta 1_{k}=\left(P_{x y}-\min \left(P_{\mathrm{La}}, P_{\mathrm{Pa}}\right)\right)_{k}, \\
& \Delta 2_{k}=\left(\max \left(P_{\mathrm{La}}, P_{\mathrm{Pa}}\right)-P_{x y}\right)_{k}, \\
& \Delta 3_{k}=\left(\tilde{P}_{x y \frac{1}{2}}-\min \left(P_{\mathrm{La}}, P_{\mathrm{Pa}}\right)\right)_{k}, \\
& \Delta 4_{k}=\left(\max \left(P_{\mathrm{La}}, P_{\mathrm{Pa}}\right)-\tilde{P}_{x y \frac{1}{2}}\right)_{k} .
\end{aligned}
$$

Selected histograms (for special values of $x$ and $y$ ) for random variables defined by (45) - (48) and for $v^{s}=v^{t}=u^{s}=u^{t}=0.1$ are presented in Figures 13, 14 and 15. The simulation results are presented in Tables 4, 5, 6 and 7.
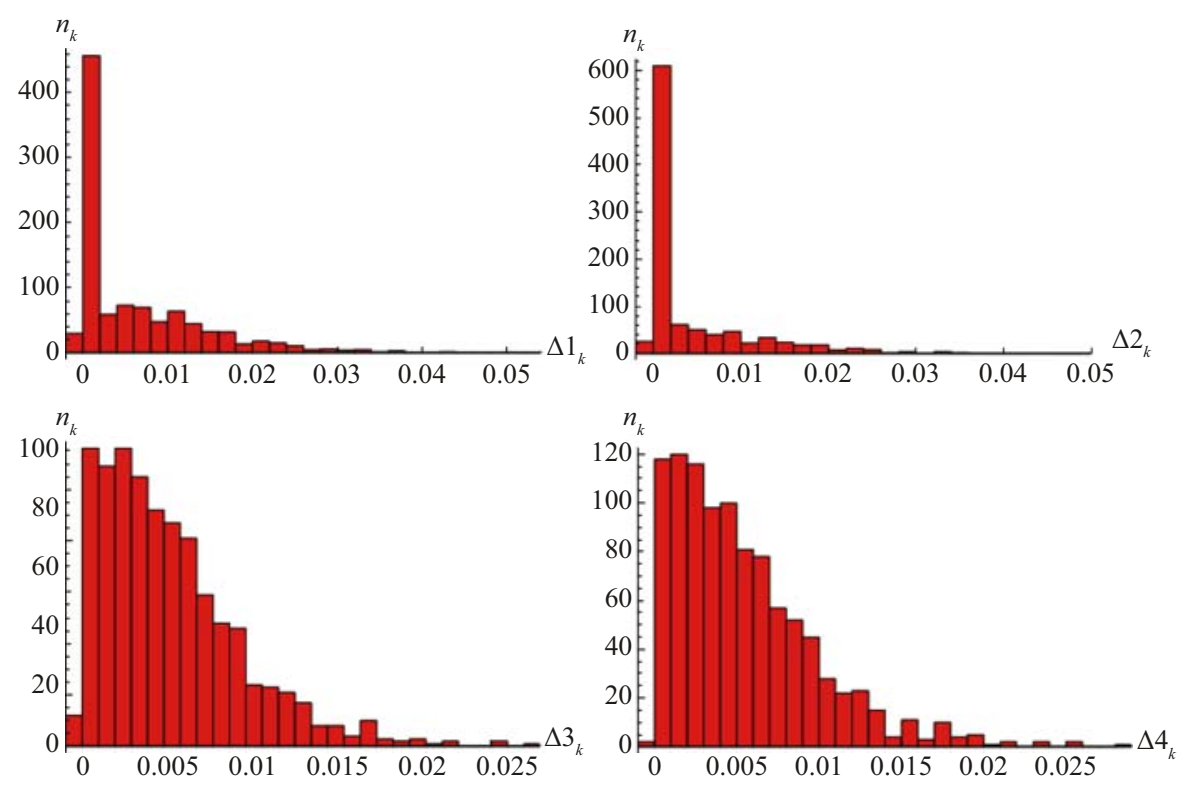

Fig. 13. Histograms for random variables $\Delta 1, \Delta 2, \Delta 3$ and $\Delta 4$ and for $x=y=0.95$. 

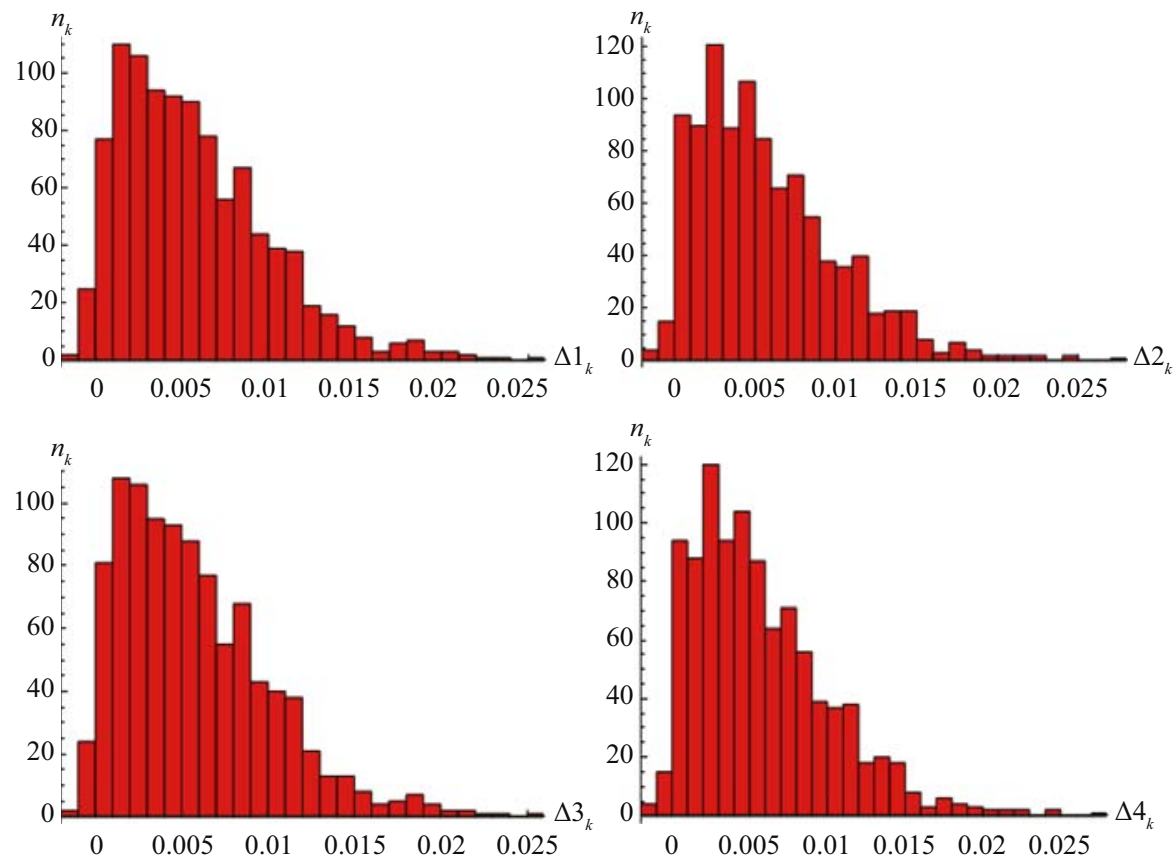

Fig. 14. Histograms for random variables $\Delta 1, \Delta 2, \Delta 3$ and $\Delta 4$ and for $x=0.6, y=0.4$.
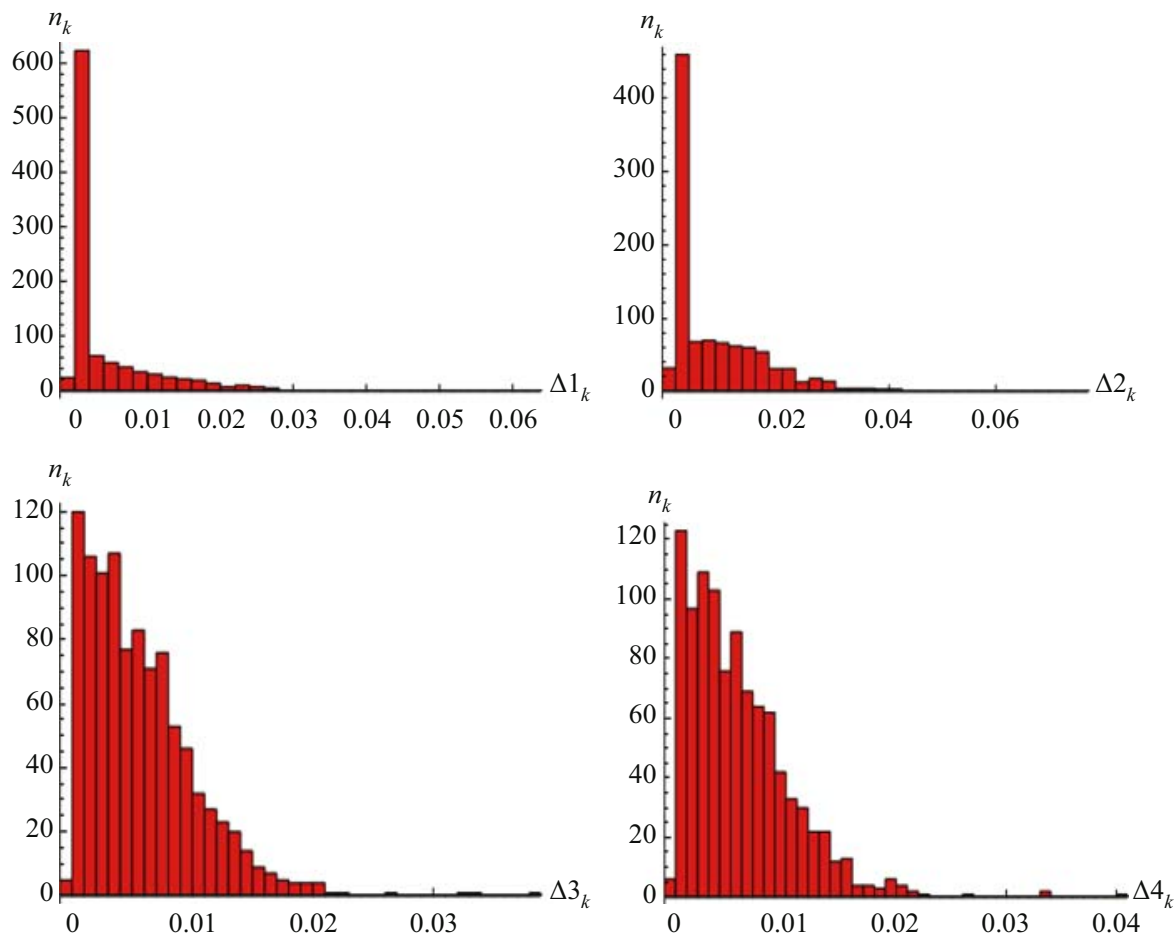

Fig. 15. Histograms for random variables $\Delta 1, \Delta 2, \Delta 3$ and $\Delta 4$ and for $x=y=0.05(*) .(*)$ Expected values of Laspeyres and Paasche formulas equal respectively: $P_{\mathrm{La}}=1.054, P_{\mathrm{Pa}}=1.044$. 
Table 4. Verifying the Laspeyres-Paasche bounding test for $P_{x y}$ and $\tilde{P}_{x y 0.5}-$ Case 1.

\begin{tabular}{|c|c|c|c|c|c|c|}
\hline \multirow[b]{2}{*}{ Statistics } & \multicolumn{6}{|c|}{ Case 1: $v^{s}=v^{t}=0.05 ; u^{s}=u^{t}=0.05$} \\
\hline & $\begin{array}{l}x=0.05 \\
y=0.05\end{array}$ & $\begin{array}{l}x=0.25 \\
y=0.25\end{array}$ & $\begin{array}{l}x=0.5 \\
y=0.5\end{array}$ & $\begin{array}{l}x=0.75 \\
y=0.75\end{array}$ & $\begin{array}{l}x=0.95 \\
y=0.05\end{array}$ & $\begin{array}{l}x=0.95 \\
y=0.95\end{array}$ \\
\hline $\begin{array}{l}\text { Mean }\left(P_{x y}\right) \\
\left(\text { Std. Dev. } P_{x y}\right)\end{array}$ & $\begin{array}{c}1.101 \\
(0.038)\end{array}$ & $\begin{array}{c}1.097 \\
(0.035)\end{array}$ & $\begin{array}{c}1.102 \\
(0.038)\end{array}$ & $\begin{array}{c}1.087 \\
(0.030)\end{array}$ & $\begin{array}{c}1.086 \\
(0.031)\end{array}$ & $\begin{array}{c}1.103 \\
(0.037)\end{array}$ \\
\hline $\begin{array}{l}\operatorname{Mean}\left(\tilde{P}_{x y 0.5}\right) \\
\left(\text { Std. Dev. } \tilde{P}_{x y 0.5}\right)\end{array}$ & $\begin{array}{c}1.103 \\
(0.038) \\
\end{array}$ & $\begin{array}{c}1.097 \\
(0.034) \\
\end{array}$ & $\begin{array}{c}1.102 \\
(0.038) \\
\end{array}$ & $\begin{array}{c}1.089 \\
(0.031) \\
\end{array}$ & $\begin{array}{c}1.086 \\
(0.031) \\
\end{array}$ & $\begin{array}{c}1.104 \\
(0.038) \\
\end{array}$ \\
\hline $\operatorname{card}\left\{k: \Delta_{1 k}<0\right\}$ & 28 & 26 & 17 & 21 & 15 & 16 \\
\hline $\operatorname{card}\left\{k: \Delta_{2 k}<0\right\}$ & 20 & 19 & 22 & 23 & 12 & 30 \\
\hline $\operatorname{card}\left\{k: \Delta_{3 k}<0\right\}$ & 7 & 25 & 17 & 15 & 15 & 2 \\
\hline $\operatorname{card}\left\{k: \Delta_{4 k}<0\right\}$ & 4 & 14 & 22 & 17 & 9 & 4 \\
\hline
\end{tabular}

Table 5. Verifying the Laspeyres-Paasche bounding test for $P_{x y}$ and $\tilde{P}_{x y 0.5}-$ Case 2.

\begin{tabular}{|l|c|c|c|c|c|c|}
\hline \multirow{2}{*}{ Statistics } & \multicolumn{7}{|c|}{ Case $2: v^{s}=v^{t}=0.2 ; u^{s}=u^{t}=0.2$} \\
\cline { 2 - 7 } & $x=0.05$ & $x=0.25$ & $x=0.5$ & $x=0.75$ & $x=0.95$ & $x=0.95$ \\
& $y=0.05$ & $y=0.25$ & $y=0.5$ & $y=0.75$ & $y=0.05$ & $y=0.95$ \\
\hline Mean $\left(P_{x y}\right)$ & 1.066 & 1.143 & 1.103 & 1.043 & 1.067 & 1.143 \\
$\left(\right.$ Std. Dev. $\left.P_{x y}\right)$ & $(0.127)$ & $(0.140)$ & $(0.126)$ & $(0.130)$ & $(0.134)$ & $(0.134)$ \\
\hline Mean $\left(\tilde{P}_{x y 0.5}\right)$ & 1.082 & 1.148 & 1.103 & 1.023 & 1.067 & 1.131 \\
$\left(\right.$ Std. Dev. $\left.\tilde{P}_{x y 0.5}\right)$ & $(0.124)$ & $(0.141)$ & $(0.126)$ & $(0.137)$ & $(0.134)$ & $(0.132)$ \\
\hline $\operatorname{card}\left\{k: \Delta_{1 k}<0\right\}$ & 28 & 28 & 39 & 44 & 21 & 34 \\
\hline $\operatorname{card}\left\{k: \Delta_{2 k}<0\right\}$ & 22 & 25 & 32 & 30 & 33 & 31 \\
\hline $\operatorname{card}\left\{k: \Delta_{3 k}<0\right\}$ & 3 & 26 & 39 & 34 & 19 & 3 \\
\hline $\operatorname{card}\left\{k: \Delta_{4 k}<0\right\}$ & 7 & 20 & 32 & 21 & 27 & 5 \\
\hline
\end{tabular}

Table 6. Verifying the Laspeyres-Paasche bounding test for $P_{x y}$ and $\tilde{P}_{x y 0.5}-$ Case 3.

\begin{tabular}{|c|c|c|c|c|c|c|}
\hline \multirow[b]{2}{*}{ Statistics } & \multicolumn{6}{|c|}{ Case 3: $v^{s}=v^{t}=0.05 ; u^{s}=u^{t}=0.2$} \\
\hline & $\begin{array}{l}x=0.05 \\
y=0.05\end{array}$ & $\begin{array}{l}x=0.25 \\
y=0.25\end{array}$ & $\begin{array}{l}x=0.5 \\
y=0.5\end{array}$ & $\begin{array}{l}x=0.75 \\
y=0.75\end{array}$ & $\begin{array}{l}x=0.95 \\
y=0.05\end{array}$ & $\begin{array}{l}x=0.95 \\
y=0.95\end{array}$ \\
\hline $\begin{array}{l}\text { Mean }\left(P_{x y}\right) \\
\left(\text { Std. Dev. } P_{x y}\right)\end{array}$ & $\begin{array}{c}1.068 \\
(0.033)\end{array}$ & $\begin{array}{c}1.092 \\
(0.034)\end{array}$ & $\begin{array}{c}1.047 \\
(0.047)\end{array}$ & $\begin{array}{c}1.107 \\
(0.039)\end{array}$ & $\begin{array}{c}1.113 \\
(0.044)\end{array}$ & $\begin{array}{c}1.110 \\
(0.040)\end{array}$ \\
\hline $\begin{array}{l}\text { Mean }\left(\tilde{P}_{x y 0.5)}\right) \\
(\text { Std. Dev.P्P } x y 0.5)\end{array}$ & $\begin{array}{c}1.070 \\
(0.032)\end{array}$ & $\begin{array}{c}1.093 \\
(0.034)\end{array}$ & $\begin{array}{c}1.047 \\
(0.047)\end{array}$ & $\begin{array}{c}1.109 \\
(0.041)\end{array}$ & $\begin{array}{c}1.113 \\
(0.044)\end{array}$ & $\begin{array}{c}1.109 \\
(0.041)\end{array}$ \\
\hline $\operatorname{card}\left\{k: \Delta_{1 k}<0\right\}$ & 24 & 27 & 34 & 40 & 24 & 28 \\
\hline $\operatorname{card}\left\{k: \Delta_{2 k}<0\right\}$ & 31 & 30 & 33 & 25 & 19 & 34 \\
\hline $\operatorname{card}\left\{k: \Delta_{3 k}<0\right\}$ & 8 & 21 & 34 & 35 & 24 & 3 \\
\hline $\operatorname{card}\left\{k: \Delta_{4 k}<0\right\}$ & 6 & 21 & 33 & 20 & 19 & 4 \\
\hline
\end{tabular}


Table 7. Verifying the Laspeyres-Paasche bounding test for $P_{x y}$ and $\tilde{P}_{x y 0.5}-$ Case 4.

\begin{tabular}{|l|c|c|c|c|c|c|}
\hline \multirow{2}{*}{ Statistics } & \multicolumn{6}{|c|}{ Case $4: v^{s}=v^{t}=0.2 ; u^{s}=u^{t}=0.05$} \\
\cline { 2 - 7 } & $x=0.05$ & $x=0.25$ & $x=0.5$ & $x=0.75$ & $x=0.95$ & $x=0.95$ \\
& $y=0.05$ & $y=0.25$ & $y=0.5$ & $y=0.75$ & $y=0.05$ & $y=0.95$ \\
\hline Mean $\left(P_{x y}\right)$ & 1.064 & 1.067 & 1.078 & 1.091 & 1.109 & 1.064 \\
$\left(\right.$ Std. Dev. $\left.P_{x y}\right)$ & $(0.128)$ & $(0.132)$ & $(0.126)$ & $(0.128)$ & $(0.128)$ & $(0.127)$ \\
\hline Mean $\left(\tilde{P}_{x y 0.5}\right)$ & 1.065 & 1.062 & 1.078 & 1.094 & 1.109 & 1.065 \\
$\left(\right.$ Std. Dev. $\left.\tilde{P}_{x y 0.5}\right)$ & $(0.125)$ & $(0.131)$ & $(0.126)$ & $(0.129)$ & $(0.128)$ & $(0.128)$ \\
\hline $\operatorname{card}\left\{k: \Delta_{1 k}<0\right\}$ & 21 & 38 & 26 & 26 & 30 & 18 \\
\hline $\operatorname{card}\left\{k: \Delta_{2 k}<0\right\}$ & 28 & 30 & 39 & 39 & 27 & 29 \\
\hline $\operatorname{card}\left\{k: \Delta_{3 k}<0\right\}$ & 2 & 24 & 26 & 22 & 25 & 4 \\
\hline $\operatorname{card}\left\{k: \Delta_{4 k}<0\right\}$ & 7 & 25 & 39 & 27 & 16 & 6 \\
\hline
\end{tabular}

\section{Simulation 2}

The presented simulation study is a continuation of the previous one but, it is done for 10000 repetitions. For the given probability distributions of prices and quantities (see Simulation 1), we observe fluctuations of the following random variables: $P_{\mathrm{La}}, P_{\mathrm{Pa}}, P_{\mathrm{F}}$, and $P_{x x}, \tilde{P}_{x x 0.5}, \tilde{P}_{x x x_{2}^{1}}^{A}$ for different values of $x$. The results for Cases $1-4$ are presented in Tables 8-11.

Table 8. Basic characteristics of the considered price indices for data from case 1.

\begin{tabular}{|l|c|c|c|c|c|c|}
\hline Statistics: & \multicolumn{6}{|c|}{ Mean / (Standard deviation) / (Volatility coefficient) for Case 1 } \\
\hline Index & $x=0.2$ & $x=0.3$ & $x=0.4$ & $x=0.6$ & $x=0.7$ & $x=0.8$ \\
\hline$P_{\mathrm{La}}$ & \multicolumn{7}{|c|}{$1.05422 /(0.03998) /(0.03792)$} \\
\hline$P_{\mathrm{Pa}}$ & \multicolumn{7}{|c|}{$1.05071 /(0.04375) /(0.04164)$} \\
\hline$P_{\mathrm{F}}$ & 1.05345 & 1.05308 & 1.05272 & 1.05202 & 1.05168 & 1.05135 \\
\hline$P_{x x}$ & $(0.04068)$ & $(0.04105)$ & $(0.04141)$ & $(0.04217)$ & $(0.04256)$ & $(0.04295)$ \\
& $(0.03862)$ & $(0.03898)$ & $(0.03934$ & $(0.04009)$ & $(0.04047)$ & $(0.04085)$ \\
& 1.05240 & 1.05238 & 1.05237 & 1.05237 & 1.05238 & 1.05240 \\
& $(0.04174)$ & $(0.04177)$ & $(0.04178)$ & $(0.04178)$ & $(0.04177)$ & $(0.04174)$ \\
& $(0.03966)$ & $(0.03969)$ & $(0.03971)$ & $(0.03971)$ & $(0.03969)$ & $(0.03966)$ \\
\hline$\tilde{P}_{x x 0.5}$ & 1.05270 & 1.05277 & 1.05281 & 1.05281 & 1.05277 & 1.05270 \\
& $(0.04141)$ & $(0.04133)$ & $(0.04129)$ & $(0.04129)$ & $(0.04133)$ & $(0.04141)$ \\
\hline$\tilde{P}_{x x 0.5}^{A}$ & $(0.03933)$ & $(0.03926)$ & $(0.03922)$ & $(0.03922)$ & $(0.03926)$ & $(0.03933)$ \\
&
\end{tabular}


Table 9. Basic characteristics of considered price indices for data from Case 2.

\begin{tabular}{|l|c|c|c|c|c|c|}
\hline Statistics: & \multicolumn{5}{|c|}{ Mean / (Standard deviation) / (Volatility coefficient) for Case 2 } \\
\hline Index & $x=0.2$ & $x=0.3$ & $x=0.4$ & $x=0.6$ & $x=0.7$ & $x=0.8$ \\
\hline$P_{\mathrm{La}}$ & \multicolumn{7}{|c|}{$1.09340 /(0.13236) /(0.12105)$} \\
\hline$P_{\mathrm{Pa}}$ & \multicolumn{7}{|c|}{$1.08288 /(0.12911) /(0.11922)$} \\
\hline$P_{\mathrm{F}}$ & \multicolumn{7}{|c|}{$/(0.12992) /(0.12114)$} \\
\hline$P_{x x}$ & 1.08928 & 1.08720 & 1.08512 & 1.08093 & 1.07882 & 1.07670 \\
& $(0.13092)$ & $(0.13036)$ & $(0.12993)$ & $(0.12941)$ & $(0.12934)$ & $(0.12940)$ \\
& $(0.12019)$ & $(0.11991)$ & $(0.11973)$ & $(0.11972)$ & $(0.11989)$ & $(0.12018)$ \\
\hline$\tilde{P}_{x x 0.5}$ & 1.08297 & 1.08300 & 1.08302 & 1.08302 & 1.08300 & 1.08297 \\
& $(0.12942)$ & $(0.12952)$ & $(0.12959)$ & $(0.12959)$ & $(0.12952)$ & $(0.12942)$ \\
& $(0.11950)$ & $(0.11960)$ & $(0.11965)$ & $(0.11965)$ & $(0.11960)$ & $(0.11950)$ \\
\hline$\tilde{P}_{x x 0.5}^{A}$ & 1.08371 & 1.08396 & 1.08411 & 1.08411 & 1.08396 & 1.08371 \\
& $(0.12934)$ & $(0.12943)$ & $(0.12948)$ & $(0.12948)$ & $(0.12943)$ & $(0.12934)$ \\
& $(0.11935)$ & $(0.11940)$ & $(0.11943)$ & $(0.11943)$ & $(0.11940)$ & $(0.11935)$ \\
\hline
\end{tabular}

Table 10. Basic characteristics of the considered price indices for data from case 3.

\begin{tabular}{|l|c|c|c|c|c|c|}
\hline Statistics: & \multicolumn{7}{|c|}{ Mean / (Standard deviation) / (Volatility coefficient) for Case 3 } \\
\hline Index & $x=0.2$ & $x=0.3$ & $x=0.4$ & $x=0.6$ & $x=0.7$ & $x=0.8$ \\
\hline$P_{\mathrm{La}}$ & \multicolumn{7}{|c|}{$1.04143 /(0.04959) /(0.04761)$} \\
\hline$P_{\mathrm{Pa}}$ & \multicolumn{7}{|c|}{$1.04597 /(0.04688) /(0.04482)$} \\
\hline$P_{\mathrm{F}}$ & \multicolumn{7}{|c|}{$/ 04477) /(0.04261)$} \\
\hline$P_{x x}$ & 1.04294 & 1.04375 & 1.04460 & 1.04641 & 1.04738 & 1.04839 \\
& $(0.04872)$ & $(0.04268)$ & $(0.04780)$ & $(0.04683)$ & $(0.04633)$ & $(0.04581)$ \\
& $(0.04671)$ & $(0.04624)$ & $(0.04576)$ & $(0.04475)$ & $(0.04423)$ & $(0.04370)$ \\
\hline$\tilde{P}_{x x 0.5}$ & 1.04566 & 1.04556 & 1.04550 & 1.04556 & 1.04550 & 1.04566 \\
& $(0.04716)$ & $(0.04725)$ & $(0.04730)$ & $(0.04730)$ & $(0.04725)$ & $(0.04716)$ \\
& $(0.04510)$ & $(0.04519)$ & $(0.04524)$ & $(0.04524)$ & $(0.04519)$ & $(0.04510)$ \\
\hline$\tilde{P}_{x x 0.5}^{A}$ & 1.04561 & 1.04550 & 1.04543 & 1.04550 & 1.04543 & 1.04561 \\
& $(0.04702)$ & $(0.04707)$ & $(0.04710)$ & $(0.04710)$ & $(0.04707)$ & $(0.04702)$ \\
& $(0.04497)$ & $(0.04502)$ & $(0.04505)$ & $(0.04505)$ & $(0.04502)$ & $(0.04497)$ \\
\hline
\end{tabular}

Table 11. Basic characteristics of the considered price indices for data from case 4.

\begin{tabular}{|l|c|c|c|c|c|c|}
\hline Statistics: & \multicolumn{5}{|c|}{ Mean / (Standard deviation) / (Volatility coefficient) for Case 4 } \\
\hline Index & $x=0.2$ & $x=0.3$ & $x=0.4$ & $x=0.6$ & $x=0.7$ & $x=0.8$ \\
\hline$P_{\mathrm{La}}$ & \multicolumn{7}{|c|}{$1.07090 /(0.12855) /(0.12004)$} \\
\hline$P_{\mathrm{Pa}}$ & \multicolumn{7}{|c|}{$1.07749 /(012567) /(0.11663)$} \\
\hline$P_{\mathrm{F}}$ & \multicolumn{7}{|c|}{$/(0.12423) /(0.11459)$} \\
\hline$P_{x x}$ & 1.07463 & 1.07627 & 1.07777 & 1.08037 & 1.08148 & 1.08247 \\
& $(0.12738)$ & $(0.12687)$ & $(0.12639)$ & $(0.12555)$ & $(0.12517)$ & $(0.12483)$ \\
& $(0.11854)$ & $(0.11788)$ & $(0.11727)$ & $(0.11621)$ & $(0.11574)$ & $(0.11532)$ \\
\hline$\tilde{P}_{x x 0.5}$ & 1.07854 & 1.07887 & 1.07907 & 1.07907 & 1.07887 & 1.07854 \\
& $(0.12585)$ & $(0.12591)$ & $(0.12594)$ & $(0.12594)$ & $(0.12591)$ & $(0.12585)$ \\
& $(0.11668)$ & $(0.11670)$ & $(0.11671)$ & $(0.11671)$ & $(0.11670)$ & $(0.11668)$ \\
\hline$\tilde{P}_{x x 0.5}^{A}$ & 1.07671 & 1.07647 & 1.07633 & 1.07633 & 1.07647 & 1.07671 \\
& $(0.12595)$ & $(0.12604)$ & $(0.12610)$ & $(0.12610)$ & $(0.12604)$ & $(0.12595)$ \\
& $(0.11698)$ & $(0.11709)$ & $(0.11715)$ & $(0.11715)$ & $(0.11709)$ & $(0.11698)$ \\
\hline
\end{tabular}




\section{Empirical Study}

As it was mentioned earlier (see Subsection 3.3), the Consumer Price Index (CPI) is commonly used as a basic measure of inflation. The index approximates changes in the costs of household consumption assuming the constant utility (COLI, Cost of Living Index). Although in practice the Laspeyres price index is used to measure the CPI, many statisticians and economists consider the Fisher index to be the best approximation of COLI. Thus, in the following section we apply $P_{x x}, \tilde{P}_{x x 0.5}$ and $\tilde{P}_{x x 0.5}^{A}$ indices to verify their
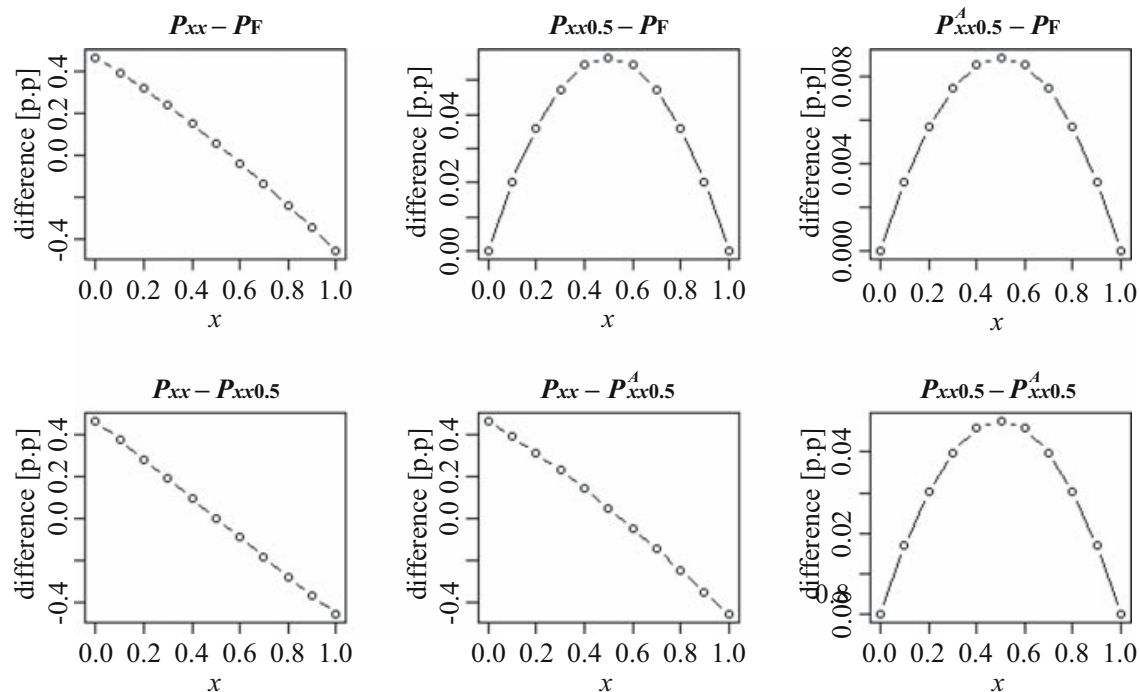

Fig. 16. Differences between indices from the considered subfamilies and the Fisher index* (Bulgaria, 2011) (*) $P_{\mathrm{La}}=1.0438, P_{\mathrm{F}}=1.0392$.
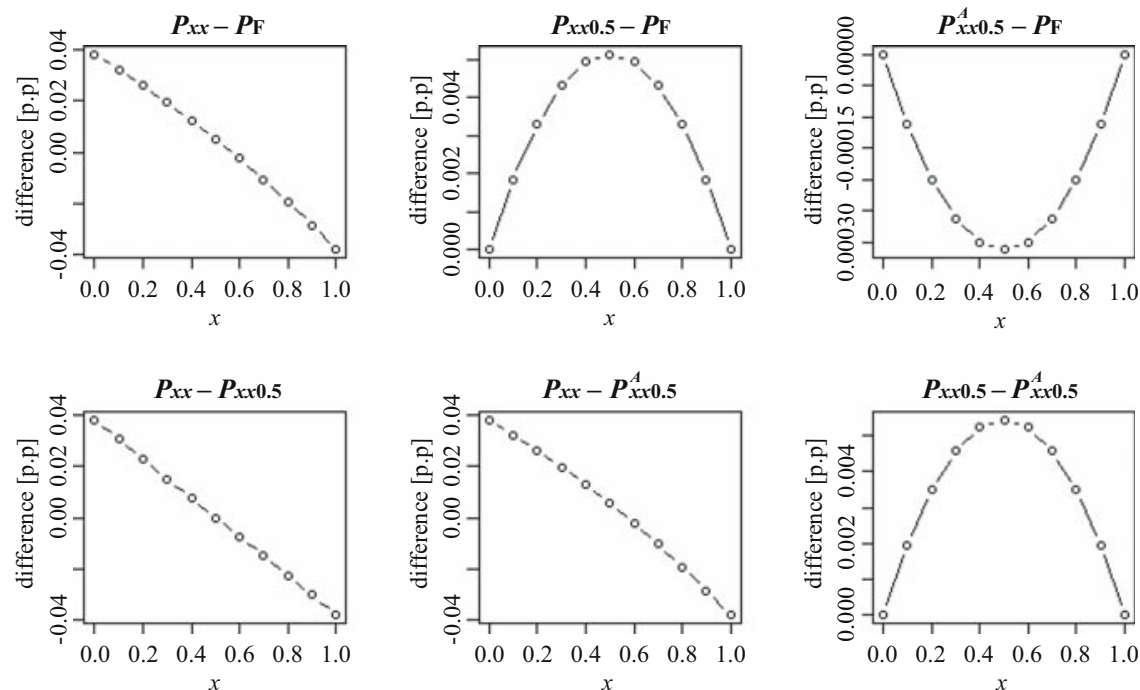

Fig. 17. Differences between indices from the considered subfamilies and the Fisher index* (Bulgaria, 2016) (*) $P_{\mathrm{La}}=0.9841, P_{\mathrm{F}}=0.9838$. 
Fisher formula approximations and distances among them using CPI data from the United Kingdom and Bulgaria. Currently there are no differences between the CPI and the HICP (Harmonised Index of Consumer Prices) in the case of these countries. Thus, we use yearly data from Eurostat from the COICOP-4 digit level of aggregation and we calculate the above-mentioned price indices for different values of $x$ and for years 2011 and 2016. The computed differences (in percentage points) between the proposed indices and the Fisher price index are presented in Figures 16-19.
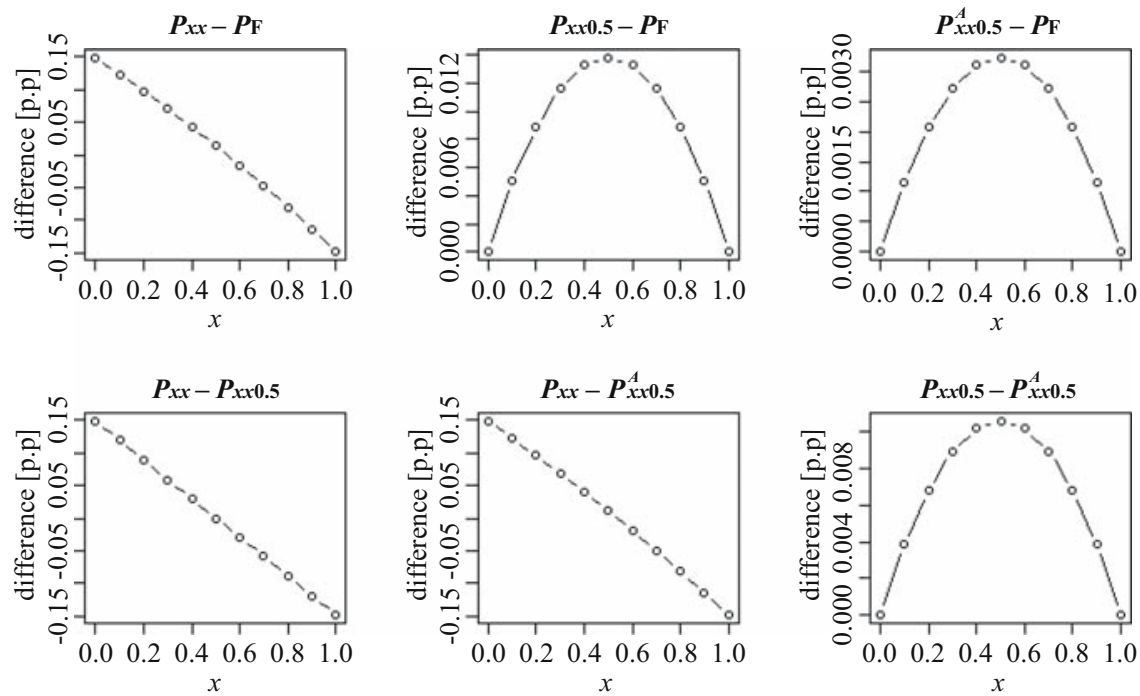

Fig. 18. Differences between indices from the considered subfamilies and the Fisher index* (United Kingdom, 2011). (*) $P_{\mathrm{La}}=1.0459, P_{\mathrm{F}}=1.0444$.
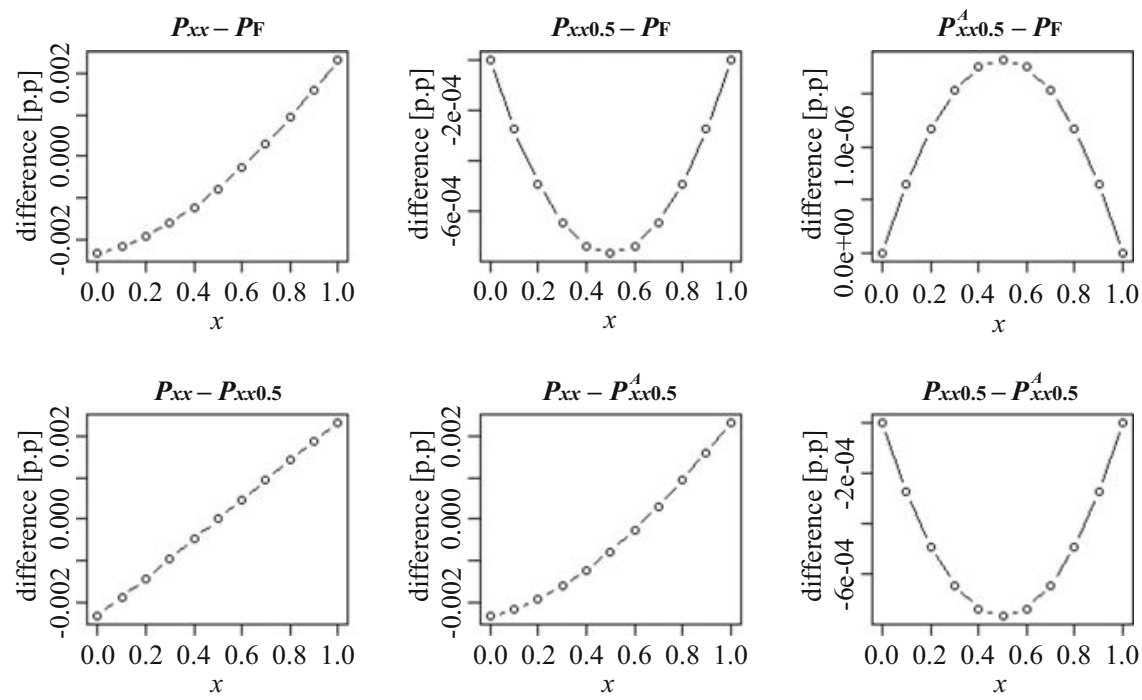

Fig. 19. Differences between indices from the considered subfamilies and the Fisher index* (United Kingdom, 2016). (*) $P_{\mathrm{La}}=0.99841, P_{\mathrm{F}}=0.99843$. 


\section{Conclusions}

In Simulation 1, we observe that indices $P_{x y}$ and $\tilde{P}_{x y 0.5}$ provide identical results for $x=y=0.5$ and quite similar results for other values of parameters $x$ and $y$, that is, we observe small differences between expected index values (arithmetic means) calculated for their generated values. These expected values are nonmonotonic functions of $x$ and $y$ hence we cannot recommend such parameter values $\left(x_{0}, y_{0}\right)$ that would lead to minimisation or maximisation of the considered general price indices $P_{x y}$ and $\tilde{P}_{x y 0.5}$. It is worth adding that values of these indices may strongly depend on parameters $x$ and $y$, that is, indices belonging to this general class of price indices may differ substantially from each other. For instance, in Case 2 (see Table 5) means of generated $P_{x y}$ values are as follows: $1.067(x=0.95, y=0.05)$ or $1.143(x=0.95, y=0.95)$ and analogical means of generated $\tilde{P}_{x y 0.5}$ values equal: $1.067(x=0.95, y=0.05)$ or $1.131(x=0.95, y=0.95)$. The precision of estimation of $P_{x y}$ and $\tilde{P}_{x y 0.5}$ indices, that is, the standard deviations of their generated values, is comparable with respect to the size of the parameters and they do not seem to depend on $x$ and $y$ (see Tables 4-7). This is a practical conclusion: even if fluctuations of prices and quantities are large, we observe similar volatility among price indices from the same general class of indices. Nevertheless, comparing results from Tables 4, 6 and 7, we can conclude that rather price fluctuations than quantity fluctuations influence volatilities of $P_{x y}$ and $\tilde{P}_{x y 0.5}$ indices. Finally, the most crucial difference between the compared general class of indices is that the probability ${ }^{1}$ of satisfying the LaspeyresPaasche bounding test is bigger in the case of $\tilde{P}_{x y 0.5}$ index (it is much bigger for small (near zero) and big (near one) values of $x$ and $y$ ). The above-mention probability is estimated as a ratio of the number of generated cases when the considered price index fulfills the Laspeyres-Paasche bounding test and the total number of repetitions. In other words, we observe relatively fewer cases when the value of $\tilde{P}_{x y 0.5}$ index is outside of the interval determined by the Laspeyres and Paasche price indices in comparison with analogical cases for the $P_{x y}$ formula (see Tables 4-7 and also Figures 13-15).

In Simulation 2, we observe that the range of expected values of $P_{x x}$ is relatively big (depending on $x$ ) in Cases 1 and 4, that is, when prices are strongly fluctuated (in Case 4 the maximum difference equals almost 0.8 p.p, see Table 11). In the same cases, expected (mean) values of generated indices from $\tilde{P}_{x x 0.5}$ and $\tilde{P}_{x x 0.5}^{A}$ classes remain stable and their changes are not bigger than 0.1 p.p (Table 8 and Table 11). Moreover, even if price fluctuations are really small (Case 3, see Table 10), generated values of $P_{x x}$ indices may differ from each other by more than 0.5 p.p. The most important fact is that although volatilities of generated indices are comparable in each case (obviously volatility coefficients are higher in Cases 1 and 4 connected with high values of price dispersions), only values of $\tilde{P}_{x x 0.5}$ and $\tilde{P}_{x x 0.5}^{A}$ indices seem to approximate the mean of generated Fisher price indices effectively. Taking into consideration also (22), (23) and (24), it may seem likely that indices from the $\tilde{P}_{x x 0.5}$ subclass are closest to superlative price indices.

The Empirical study confirms previously obtained results. Indices from the $\tilde{P}_{x x 0.5}$ and $\tilde{P}_{x x 0.5}^{A}$ subfamilies generate values that are very close to the superlative Fisher index and differences between them are very small. When the effect of substitution is observed, that is, when the difference between values of Laspeyres and Paasche indices rises, we can note 
large differences between $P_{x x}$ indices and the Fisher index, and between $P_{x x}$ indices and indices from the $\tilde{P}_{x x 0.5}$ and $\tilde{P}_{x x 0.5}^{A}$ subfamilies (see Figures 16 and 18). When the CPI has no substitution bias $\left(P_{\mathrm{La}} \approx P_{\mathrm{F}}\right)$, the values of indices from all the considered subfamilies approximate each other (see Figures 17 and 19). And one more remark - only the differences $P_{x x}-P_{\mathrm{F}}, P_{x x}-\tilde{P}_{x x 0.5}$ and $P_{x x}-\tilde{P}_{x x 0.5}^{A}$, as functions of $x \in[0,1]$, seem to be monotonic and approximately linear.

\subsection{Final Remarks}

The proposed and wide class of price indices $\left(\tilde{P}_{x y z}\right)$ has similar axiomatic properties as the geo-logarithmic price index family and, in particular, each index from this family satisfies the Martini's minimal requirements. It is worth adding that cofactors of $\tilde{P}_{x y z}$ indices satisfy the proportionality and homogeneity axioms (see Subsection 4.3). It is important from the perspective of the economic approach that there is a possibility of modification of the $\tilde{P}_{x x z}$ family to obtain such a general class of indices $\left(\tilde{P}_{x x z}^{A}\right)$ that satisfies the Laspeyres-Paasche bounding test (Theorem 4). It should also be added that the particular element of the $\tilde{P}_{x y z}$ and $\tilde{P}_{x x z}^{A}$ families is the superlative Fisher price index, which is not an element of the geologarithmic price index class. Moreover, for any value of $x \in[0,1]$ generated $\tilde{P}_{x x 0.5}$ and $\tilde{P}_{x x 0.5}^{A}$ indices seem to approximate the values of the Fisher price indices effectively. Thus, since for the superlative Walsh and Fisher price indices it holds that $\tilde{P}_{\frac{1}{2}} \frac{1}{2} \frac{1}{2}=P_{\mathrm{W}}$ and $\tilde{P}_{00 \frac{1}{2}}$ $\tilde{P}_{11 \frac{1}{2}}=P_{\mathrm{F}}$, the subfamily $\tilde{P}_{x x \frac{1}{2}}$ seems to be worth further studying. From the theoretical point of view, it would be interesting to consider an "average representative" of the abovementioned subclass of indices, that is, the price index calculated for some $x_{0}$ which fulfils $\tilde{P}_{x_{0} x_{0} \frac{1}{2}}=\int_{0}^{1} \tilde{P}_{x x \frac{1}{2}} d x$.

\section{Appendix}

\subsection{Appendix A}

Below we present formal definitions of major postulates (tests) arising from the axiomatic index theory and used in Theorem 1. Let us consider the price index formula $P\left(q^{s}, q^{t}, p^{s}, p^{t}\right)$. Let us also denote by $\lambda$ any $N \times N$ diagonal matrix with positive elements $\lambda_{1}, \lambda_{2}, \ldots, \lambda_{N}$ and by $k$ a positive, real number.

- Identity means that

$$
P\left(q^{s}, q^{t}, p^{s}, p^{s}\right)=1
$$

- Proportionality can be described by the following condition:

$$
P\left(q^{s}, q^{t}, p^{s}, k p^{s}\right)=k .
$$

- Commensurability can be expressed as follows:

$$
P\left(\lambda^{-1} q^{s}, \lambda^{-1} q^{t}, \lambda p^{s}, \lambda p^{t}\right)=P\left(q^{s}, q^{t}, p^{s}, p^{t}\right) .
$$


- Linear homogeneity has the following form:

$$
P\left(q^{s}, q^{t}, p^{s}, k p^{t}\right)=k P\left(q^{s}, q^{t}, p^{s}, p^{t}\right) .
$$

- Price dimensionality can be expressed as follows:

$$
P\left(q^{s}, q^{t}, k p^{s}, k p^{t}\right)=P\left(q^{s}, q^{t}, p^{s}, p^{t}\right) .
$$

- Strict monotonicity is defined as follows:

$$
P\left(q^{s}, q^{t}, p^{s}, \tilde{p}^{t}\right)>P\left(q^{s}, q^{t}, p^{s}, p^{t}\right), \quad \text { if } \quad \tilde{p}^{t} \geq p^{t}
$$

and

$$
P\left(q^{s}, q^{t}, \tilde{p}^{s}, p^{t}\right)<P\left(q^{s}, q^{t}, p^{s}, p^{t}\right), \quad \text { if } \quad \tilde{p}^{s} \geq p^{s},
$$

where $\tilde{p}^{t} \geq p^{t}$ means that at least one element of the nonnegative vector $\tilde{p}^{t}$ is greater than the corresponding element of the vector $p^{t}$ (the relation $\tilde{p}^{t} \leq p^{t}$ is defined analogously).

- Basis reversibility means that

$$
P\left(q^{s}, q^{t}, p^{s}, p^{t}\right) P\left(q^{t}, q^{s}, p^{t}, p^{s}\right)=1 .
$$

- Factor reversibility can be expressed as

$$
P\left(q^{s}, q^{t}, p^{s}, p^{t}\right) P\left(p^{s}, p^{t}, q^{s}, q^{t}\right)=\frac{\sum_{i=1}^{N} p_{i}^{t} q_{i}^{t}}{\sum_{i=1}^{N} p_{i}^{s} q_{i}^{s}} .
$$

\subsection{Appendix $B$}

Observation. Let us assume that two price indices $P_{1}$ and $P_{2}$ satisfy the axioms from the Martini's minimal requirements. Then, the price index being the weighted geometric mean of $P_{1}$ and $P_{2}$ indices, i.e., $P=P_{1}^{z} P_{2}^{1-z}$ for $z \in[0,1]$, also satisfies the Martini's minimal requirements.

Proof Let us assume that $P_{1}\left(q^{s}, q^{t}, p^{s}, p^{t}\right)$ and $P_{2}\left(q^{s}, q^{t}, p^{s}, p^{t}\right)$ satisfy identity, commensurability and linear homogeneity (see Appendix A, Subsection 8.1). Let us consider the price index $P\left(q^{s}, q^{t}, p^{s}, p^{t}\right)=P_{1}^{z}\left(q^{s}, q^{t}, p^{s}, p^{t}\right) P_{2}^{1-z}\left(q^{s}, q^{t}, p^{s}, p^{t}\right)$ for a real number $z \in[0,1]$.

The price index $P\left(q^{s}, q^{t}, p^{s}, p^{t}\right)$ also satisfies:

- Identity since $P\left(q^{s}, q^{t}, p^{s}, p^{s}\right)=P_{1}^{z}\left(q^{s}, q^{t}, p^{s}, p^{s}\right) P_{2}^{1-z}\left(q^{s}, q^{t}, p^{s}, p^{s}\right)=1^{z} 1^{1-z}=1$;

- Commensurability since for any $N \times N$ diagonal matrix $\lambda$ with positive elements $\lambda_{1}, \lambda_{2}, \ldots, \lambda_{N}$ we have

$$
\begin{aligned}
P\left(\lambda^{-1} q^{s}, \lambda^{-1} q^{t}, \lambda p^{s}, \lambda p^{t}\right) & =P_{1}^{z}\left(\lambda^{-1} q^{s}, \lambda^{-1} q^{t}, \lambda p^{s}, \lambda p^{t}\right) P_{2}^{1-z}\left(\lambda^{-1} q^{s}, \lambda^{-1} q^{t}, \lambda p^{s}, \lambda p^{t}\right) \\
& =P_{1}^{z}\left(q^{s}, q^{t}, p^{s}, p^{t}\right) P_{2}^{1-z}\left(q^{s}, q^{t}, p^{s}, p^{t}\right)=P\left(q^{s}, q^{t}, p^{s}, p^{t}\right)
\end{aligned}
$$

- Linear homogeneity

$$
\begin{aligned}
P\left(q^{s}, q^{t}, p^{s}, k p^{t}\right) & =P_{1}^{z}\left(q^{s}, q^{t}, p^{s}, k p^{t}\right) P_{2}^{1-z}\left(q^{s}, q^{t}, p^{s}, k p^{t}\right) \\
& =k^{z} P_{1}^{z}\left(q^{s}, q^{t}, p^{s}, p^{t}\right) k^{1-z} P_{2}^{1-z}\left(q^{s}, q^{t}, p^{s}, p^{t}\right)=k P\left(q^{s}, q^{t}, p^{s}, p^{t}\right) .
\end{aligned}
$$


Thus, the price index $P\left(q^{s}, q^{t}, p^{s}, p^{t}\right)$ satisfies the axioms from the system of minimal requirements proposed by Marco Martini (1992b).

\subsection{Appendix C. Heuristic Proof of Approximation (24)}

Let us note that assuming $\forall i \in\{1,2, \ldots, N\} q_{s i} \approx q_{t i}$ we obtain as a consequence

$$
q_{s i}^{x} \approx q_{t i}^{x}, \quad \text { for } \quad x \in[0,1]
$$

and thus

$$
q_{i}^{A x} \approx q_{s i}, \quad q_{i}^{B x} \approx q_{s i}, \quad \text { for } \quad i \in\{1,2, \ldots, N\}
$$

From (C2) we obtain the following approximations

$$
w_{s i}^{A x} \approx w_{s i}^{0} \text { and } w_{s i}^{B x} \approx w_{s i}^{0} .
$$

Analogically, we can write that

$$
w_{t i}^{A x} \approx w_{t i}^{1} \text { and } w_{t i}^{B x} \approx w_{t i}^{1} .
$$

Repeating steps $(\mathrm{C} 2)-(\mathrm{C} 4)$ for the approximation $q_{s i}^{y} \approx q_{t i}^{y}$ we obtain

$$
w_{s i}^{A y} \approx w_{s i}^{0}, \quad w_{s i}^{B y} \approx w_{s i}^{0}, \quad w_{t i}^{A y} \approx w_{t i}^{1}, \quad w_{t i}^{B y} \approx w_{t i}^{1} .
$$

It is proved by Fattore (2010) that within the limit $w_{s i}^{x} \rightarrow w_{t i}^{y}$ it holds that

$$
P_{x y}=\prod_{i=1}^{N}\left(\frac{p_{t i}}{p_{s i}}\right)^{v_{i}^{x y}} \approx \prod_{i=1}^{N}\left(\frac{p_{t i}}{p_{s i}}\right)^{\frac{w_{s i}^{x}+w_{t i}^{y}}{2}} .
$$

Since $w_{s i}^{x}=w_{s i}^{A x}, w_{t i}^{y}=w_{t i}^{A y}$ and consequently $v_{i}^{x y}=v_{A i}^{x y}$ from (C6) and the assumption that $w_{s i}^{x} \approx w_{t i}^{y}$ we have

$$
\prod_{i=1}^{N}\left(\frac{p_{t i}}{p_{s i}}\right)^{v_{A i}^{x y}} \approx \prod_{i=1}^{N}\left(\frac{p_{t i}}{p_{s i}}\right)^{\frac{w_{s i}^{A x}+w_{t i}^{A y}}{2}}
$$

Analogically to the Fattore's way, it can be proved that

$$
\prod_{i=1}^{N}\left(\frac{p_{t i}}{p_{s i}}\right)^{v_{B i}^{x y}} \approx \prod_{i=1}^{N}\left(\frac{p_{t i}}{p_{s i}}\right)^{\frac{w_{s i}^{B x}+w_{t i}^{B y}}{2}}
$$

Thus, from (18), (C7) and (C8) we obtain

$$
\begin{aligned}
\tilde{P}_{x y z} & =\left\{\prod_{i=1}^{N}\left(\frac{p_{t i}}{p_{s i}}\right)^{v_{A i}^{x y}}\right\}^{z}\left\{\prod_{i=1}^{N}\left(\frac{p_{t i}}{p_{s i}}\right)^{v_{B i}^{x y}}\right\}^{1-z} \\
& \approx \prod_{i=1}^{N}\left(\frac{p_{t i}}{p_{s i}}\right)^{\frac{1}{2}\left\{z\left(w_{s i}^{A x}+w_{t i}^{A y}\right)+(1-z)\left(w_{s i}^{B x}+w_{t i}^{B y}\right)\right\}} .
\end{aligned}
$$


From (C3), (C4), (C5) and (C9) we obtain the final conclusion that

$$
\tilde{P}_{x y z} \approx \prod_{i=1}^{N}\left(\frac{p_{t i}}{p_{s i}}\right)^{\frac{w_{s i}^{0}+w_{t i}^{1}}{2}}=P_{\mathrm{T}}
$$

\subsection{Appendix D (Proof of Theorem 4)}

Lemma For any positive real values $a, b, c, d$ and $x \in[0,1]$ the following relation is true

$$
\min \left\{\frac{a}{c}, \frac{b}{d}\right\} \leq \frac{a x+b(1-x)}{c x+d(1-x)} \leq \max \left\{\frac{a}{c}, \frac{b}{d}\right\}
$$

\section{Proof of the Lemma}

Let us note that in the case of $x=0$ or $x=1$ the relation (D1) is obvious. Let us consider $x \in(0,1)$ and, for instance, let us assume that

$$
\frac{a}{c} \leq \frac{b}{d}
$$

Suppose by contraposition that (D1) does not hold, that is, there exists some $x_{0} \in(0,1)$ that

$$
\frac{a x_{0}+b\left(1-x_{0}\right)}{c x_{0}+d\left(1-x_{0}\right)}<\frac{a}{c}
$$

The inequality (D3) can be written equivalently as

$$
a c x_{0}+b c\left(1-x_{0}\right)<a c x_{0}+a d\left(1-x_{0}\right),
$$

and that leads to the false (with respect to the assumption (D2)) conclusion that

$$
\frac{b}{d}<\frac{a}{c}
$$

In an analogous way, we can prove that under the assumption (D2) it is impossible to obtain

$$
\frac{a x_{0}+b\left(1-x_{0}\right)}{c x_{0}+d\left(1-x_{0}\right)}>\frac{b}{d} \text {. }
$$

\section{Proof of Theorem 4}

Firstly, let us consider any $(x, z) \in(0,1) \times(0,1)$. Let us signify by

$$
\begin{aligned}
& \theta_{1}(x)=\sum_{i=1}^{N} p_{t i}\left(x q_{t i}+(1-x) q_{s i}\right), \\
& \theta_{2}(x)=\sum_{i=1}^{N} p_{s i}\left(x q_{t i}+(1-x) q_{s i}\right), \\
& \theta_{3}(x)=\sum_{i=1}^{N} p_{t i}\left((1-x) q_{t i}+x q_{s i}\right),
\end{aligned}
$$




$$
\theta_{4}(x)=\sum_{i=1}^{N} p_{s i}\left((1-x) q_{t i}+x q_{s i}\right)
$$

From (28) and (D7)-(D10) we have that

$$
\ln \left(\tilde{P}_{x x z}^{A}\right)=z\left(\ln \theta_{1}(x)-\ln \left(\theta_{2}(x)\right)+(1-z)\left(\ln \theta_{3}(x)-\ln \left(\theta_{4}(x)\right),\right.\right.
$$

and thus, according to the necessary condition for the existence of the local extreme, it must hold

$$
\frac{\partial \ln \left(\tilde{P}_{x x z}^{A}\right)}{\partial z}=\ln \frac{\theta_{1}(x) \theta_{4}(x)}{\theta_{2}(x) \theta_{3}(x)}=0
$$

From (D6) we obtain immediately that

$$
\theta_{1}(x) \theta_{4}(x)=\theta_{2}(x) \theta_{3}(x)
$$

and it leads to the following condition

$$
\sum_{i=1}^{N} p_{t i} q_{t i} \sum_{i=1}^{N} p_{s i} q_{s i}\left[x^{2}-(1-x)^{2}\right]=\sum_{i=1}^{N} p_{t i} q_{s i} \sum_{i=1}^{N} p_{s i} q_{t i}\left[x^{2}-(1-x)^{2}\right]
$$

Since in (D14) we take into consideration any prices and quantities, we conclude that it must hold that $x^{2}-(1-x)^{2}=0$ or equivalently $x=0.5$. Let us note that taking $x=\frac{1}{2}$ we obtain $\tilde{P}_{\frac{1}{2} \frac{1}{2} z}^{A}=P_{\mathrm{ME}}$ and this formula does not depend on the parameter $z$. In other words, since it holds that

$$
\frac{\partial \ln \left(\tilde{P}_{0.50 .5 z}^{A}\right)}{\partial z}=0
$$

that is, each point on the plane $(0.5, z)$ is a stationary point for the function $\ln \left(\tilde{P}_{x x z}^{A}\right)$ (and thus, also for $\left.\tilde{P}_{x x z}^{A}\right)$ depending on $(x, z)$. Thus, the potential local extreme of the function $\tilde{P}_{x x z}^{A}$ is obtained in such points and it equals $P_{\mathrm{ME}}$.

Now, let us verify the behaviour of the function $\tilde{P}_{x x z}^{A}$ in the frontier of the set $[0,1] \times[0,1]$, here denoted by $D=\operatorname{Fr}([0,1] \times[0,1])$. To reach this purpose, let us consider the following sets: $D_{1}=\{(x, z): x \in\{0,1\} \wedge z \in(0,1)\}, D_{2}=\{(x, z)$ : $x \in(0,1) \wedge z \in\{0,1\}\}, \quad D_{3}=\{(0,0),(0,1),(1,0),(1,1)\}$, where obviously $D=$ $D_{1} \cup D_{2} \cup D_{3}$. Let us note that limiting the domain of the function $\tilde{P}_{x x z}^{A}$ to $D_{1}$ we obtain for $z \in(0,1)$

$$
\tilde{P}_{00 z}^{A}=P_{\mathrm{La}}^{z} P_{\mathrm{Pa}}^{1-z} \quad \text { or } \quad \tilde{P}_{11 z}^{A}=P_{\mathrm{La}}^{1-z} P_{\mathrm{Pa}}^{z}
$$

where obviously the price index being the geometric mean of the Laspeyres and Paasche price indices fulfils the Laspeyres-Paasche bounding test. Limiting the domain of the 
function $\tilde{P}_{x x z}^{A}$ to $D_{2}$ we obtain for $x \in(0,1)$

$$
\tilde{P}_{x x 0}^{A}=\frac{\sum_{i=1}^{N} p_{t i}\left((1-x) q_{t i}+x q_{s i}\right)}{\sum_{i=1}^{N} p_{s i}\left((1-x) q_{t i}+x q_{s i}\right)}
$$

or

$$
\tilde{P}_{x x 1}^{A}=\frac{\sum_{i=1}^{N} p_{t i}\left(x q_{t i}+(1-x) q_{s i}\right)}{\sum_{i=1}^{N} p_{s i}\left(x q_{t i}+(1-x) q_{s i}\right)} .
$$

For instance, taking $a=\sum_{i=1}^{N} p_{t i} q_{t i}, \quad b=\sum_{i=1}^{N} p_{t i} q_{s i}, \quad c=\sum_{i=1}^{N} p_{s i} q_{t i}$ and $d=$ $\sum_{i=1}^{N} p_{s i} q_{s i}$ from the Lemma, we have the immediate conclusion that for any $x \in(0,1)$ it holds that

$$
\min \left(P_{\mathrm{La}}, P_{\mathrm{Pa}}\right) \leq \tilde{P}_{x x 1}^{A} \leq \max \left(P_{\mathrm{La}}, P_{\mathrm{Pa}}\right),
$$

since $P_{\mathrm{La}}=\frac{b}{d}$ and $P_{\mathrm{Pa}}=\frac{a}{c}$. The analogous conclusion from the Lemma is that

$$
\min \left(P_{\mathrm{La}}, P_{\mathrm{Pa}}\right) \leq \tilde{P}_{x x 0}^{A} \leq \max \left(P_{\mathrm{La}}, P_{\mathrm{Pa}}\right),
$$

and thus, similarly to (D16), limiting the domain of the function $\tilde{P}_{x x z}^{A}$ to $D_{2}$, we can write that

$$
\tilde{P}_{x x z}^{A}=P_{\mathrm{La}}^{1-z_{0}} P_{\mathrm{Pa}}^{z_{0}},
$$

for $x \in(0,1), z \in\{0,1\}$, and some $z_{0} \in[0,1]$.

Limiting the domain of the function $\tilde{P}_{x x z}^{A}$ to $D_{3}$, from (32) and (33) we can reduce the set of the function values to $\left\{P_{\mathrm{La}}, P_{\mathrm{Pa}}\right\}$, i.e.,

$$
\left\{\tilde{P}_{x x z}^{A}:(x, z) \in D_{3}\right\}=\left\{P_{\mathrm{La}}, P_{\mathrm{Pa}}\right\} .
$$

Let us note that the function $\tilde{P}_{x x z}^{A}$ is continuous in the closed and bounded set $[0,1] \times$ $[0,1]$ being a convex quadrangle. From (D15), (D16), (D21), (D22) and the Weierstrass extreme value theorem, we know that the minimum and maximum value of the function $\tilde{P}_{x x z}^{A}$ belongs to the following set: $\left\{P_{\mathrm{La}}, P_{\mathrm{Pa}}, P_{\mathrm{La}}^{z} P_{\mathrm{Pa}}^{1-z}, P_{\mathrm{La}}^{1-z} P_{\mathrm{Pa}}^{z}, P_{\mathrm{ME}}\right\}$ for a $z \in[0,1]$. Since the price index $P_{\mathrm{ME}}$ satisfies the Laspeyres-Paasche bounding test (it is an immediate consequence of the Lemma used for $x=0.5$ and $a=\sum_{i=1}^{N} p_{t i} q_{t i}, b=\sum_{i=1}^{N} p_{t i} q_{s i}, c=$ $\sum_{i=1}^{N} p_{s i} q_{t i}$ and $d=\sum_{i=1}^{N} p_{s i} q_{s i}$.), we have the final conclusion that the above-mentioned test is also satisfied in the case of any price index from the $\tilde{P}_{x x z}^{A}$ subfamily.

\section{References}

Allen, R.G.D. 1975. Index Numbers in Theory and Practice. London: Macmillan Press.

Balk, B.M. 1995. "Axiomatic Price Index Theory: A Survey." International Statistical Review 63: 69-93. Doi: https://doi.org/10.2307/1403778.

Białek, J. 2012. "Proposition of the general formula for price indices." Communications in Statistics: Theory and Methods 41(5): 943-952. Doi: https://doi.org/10.1080/ 03610926.2010 .533238 . 
Boskin, M.J., E.R. Dulberger, R.J. Gordon, Z. Griliches, and D. Jorgenson. 1996. Toward a More Accurate Measure of the Cost of Living. Final Report to the Senate Finance Committee from the Advisory Commission to Study the Consumer Price Index.

Carlson, B.C. 1972. The logarithmic mean, Amer. Math. Monthly. 79: 615-618. Doi: https://doi.org/10.1080/00029890.1972.11993095.

Clements, K.W. and H.Y. Izan. 1987. "The Measurement of Inflation: A Stochastic Approach." Journal of Business and Economic Statistics 5: 339-350. Doi: https://doi.org/10.1080/07350015.1987.10509598.

Diewert, W.E. 1976. "Exact and Superlative Index Numbers." Journal of Econometrics 4: 114-145. Doi: https://doi.org/10.1016/0304-4076(76)90009-9.

Diewert, W.E. 1993. The economic theory of index numbers: a survey, Essays in index number theory, vol. 1, Eds. W.E. Diewert and A.O. Nakamura: 177-221, Amsterdam.

Dumagan, J. 2002. "Comparing the Superlative Törnqvist and Fisher ideal indices." Economic Letters 76: 251-258. Doi: https://doi.org/10.1016/s0165-1765(02)00049-6.

Eichhorn, W. and J. Voeller. 1976. Theory of the Price Index. Fisher's Test Approach and Generalizations. New York: Springer-Verlag.

Fattore, M. 2006. On the monotonicity of the geo-logarithmic price indexes. In: Proceedings of the XLIII Scientific Meeting, Società Italiana di Statistica, Cleup, Padova.

Fattore, M. 2010. “Axiomatic Properties of Geo-Logarithmic Price Indices.” Journal of Econometrics 156(2): 344-353. Doi: https://doi.org/10.1016/j.jeconom.2009.11.004.

Hill, R.J. 2006. "Superlative Index Numbers: Not All of Them Are Super." Journal of Econometrics 130: 25-43. Doi: https://doi.org/10.1016/j.jeconom.2004.08.018.

IMF. 2004. Producer Price Index Manual, International Monetary Fund.

Jorgenson, D.W. and D.T. Slesnick. 1983. Individual and social cost of living indexes, Price level measurement: proceedings of a conference sponsored by Statistics Canada, W.E. Diewert and C. Montmarquette: 241-336, Ottawa: Statistics Canada.

Krstcha, M. 1988. Axiomatic characterization of statistical price indices. Heidelberg: Physica-Verlag.

Martini, M. 1992a. I numeri indice in un approccio assiomatico. Giuffré, Milan.

Martini, M. 1992b. "General Function of Axiomatic Index Numbers." J. Ital. Statist. Soc 3: 359-376. Doi: https://doi.org/10.1007/bf02589086.

Olt, B. 1996. Axiom und Struktur in der statistischen Preisindextheorie. Germany: Peter Lang.

Pollak, R.A. 1989. The theory of the cost-of-living index. Oxford: Oxford University Press.

White, A.G. 1999. "Measurement Biases in Consumer Price Indexes." International

Statistical Review 3: 301-325. Doi: https://doi.org/10.1111/j.1751-5823.1999. tb00451.x.

Von der Lippe, P. 2007. Index Theory and Price Statistics. Germany: Peter Lang.

Received February 2017

Revised August 2018

Accepted September 2018 